\title{
Towards a natural classification of Dothidotthia and Thyrostroma in Dothidotthiaceae (Pleosporineae, Pleosporales)
}

\author{
Senwanna $C^{1,2,3}$, Wanasinghe $\mathrm{DN}^{1,3,4}$, Bulgakov $\mathrm{TS}^{5}$, Wang $\mathrm{Y}^{6}$, Bhat $\mathrm{DJ}^{7}$, \\ Tang $\mathrm{AMC}^{8}$, Mortimer PE ${ }^{1}$, Xu J ${ }^{1,4}$, Hyde $\mathrm{KD}^{1,3,4}$ and Phookamsak $\mathrm{R}^{1,3,4^{*}}$
}

\footnotetext{
${ }^{1}$ Key Laboratory for Plant Diversity and Biogeography of East Asia, Kunming Institute of Botany, Chinese Academy of Sciences, Kunming 650201, Yunnan, People's Republic of China

${ }^{2}$ Department of Entomology and Plant Pathology, Faculty of Agriculture, Chiang Mai University, Chiang Mai 50200, Thailand

${ }^{3}$ Center of Excellence in Fungal Research, Mae Fah Luang University, Chiang Rai 57100, Thailand

${ }^{4}$ World Agroforestry Centre, East and Central Asia, Heilongtan, Kunming 650201, Yunnan, People's Republic of China

${ }^{5}$ Russian Research Institute of Floriculture and Subtropical Crops, Yana Fabritsiusa Street, 2/28, Sochi 354002, Krasnodar Region, Russia

${ }^{6}$ Department of Plant Pathology, College of Agriculture, Guizhou University, Guiyang, Guizhou 550025, China

${ }^{7}$ No. 128/1-J, Azad Housing Society, Curca, P.O. Goa Velha-403108, India

${ }^{8}$ Division of Applied Science, College of International Education, The Hong Kong Baptist University, Hong Kong, People's Republic of China
}

Senwanna C, Wanasinghe DN, Bulgakov TS, Wang Y, Bhat DJ, Tang AMC, Mortimer PE, Xu J, Hyde KD, Phookamsak R 2019 - Towards a natural classification of Dothidotthia and Thyrostroma in Dothidotthiaceae (Pleosporineae, Pleosporales). Mycosphere 10(1), 701-738, Doi 10.5943/mycosphere/10/1/15

\begin{abstract}
Dothidotthia and Thyrostroma (Dothidotthiaceae, Pleosporineae, Pleosporales) species are plant pathogens causing canker, dieback and leaf spots on a wide range of hosts. However, the naming species is difficult, due to insufficient protologues, poor phylogenetic understanding due to the lack of sequence data from type species and low-quality illustrations. Moreover, the connections between asexual and sexual morphs of these genera are unclear. In this study, fresh samples of Dothidotthia and Thyrostroma were collected from symptomatic twigs and branches in southern European Russia. Multi-gene phylogenetic analyses based on a concatenated LSU, SSU, ITS and TEF1- $\alpha$ sequence dataset were used to investigate the phylogenetic position and confirm relationships of the asexual and sexual morphs in these genera of Dothidotthiaceae. In this study, Dothidotthia can easily be distinguished from Thyrostroma based on multi-gene phylogenetic analyses coupled with morphological characters. The new species, Dothidotthia robiniae, Thyrostroma celtidis, T. lycii, T. moricola, T. robiniae, T. styphnolobii, T. tiliae, T. ulmicola and T. ulmigenum are introduced. In addition, Neodothidotthia negundinicola clusters with species of Dothidotthia and hence Neodothidotthia is synonymized under Dothidotthia. Two new combinations, $D$. negundinicola and $D$. negundinis, are introduced.
\end{abstract}

Key words - 9 new species - Ascomycota - Dothideomycetes - Holomorph - Phylogeny Taxonomy 


\section{Introduction}

Pleosporineae M.E. Barr is a suborder of Pleosporales Luttr. ex M.E. Barr which includes 22 families, viz. Acrocalymmaceae Crous \& Trakun., Ascocylindricaceae Abdel-Wahab, Bahkali, E.B.G. Jones, Ariyaw. \& K.D. Hyde, Camarosporiaceae Wanas., Wijayaw., K.D. Hyde \& Crous, Camarosporidiellaceae Wanas., Wijayaw., Crous \& K.D. Hyde, Coniothyriaceae W.B. Cooke, Cucurbitariaceae G. Winter, Didymellaceae Gruyter, Aveskamp \& Verkley, Dothidotthiaceae Crous \& A.J.L. Phillips, Halojulellaceae Suetrong, K.D. Hyde \& E.B.G. Jones, Leptosphaeriaceae M.E. Barr, Libertasomycetaceae Crous, Microsphaeropsidaceae Qian Chen, L. Cai \& Crous, Neocamarosporiaceae Wanas., Wijayaw., Crous \& K.D. Hyde, Neophaeosphaeriaceae Ariyaw. \& K.D. Hyde, Neopyrenochaetaceae Valenz.-Lopez, Crous, Cano, Guarro \& Stchigel, Parapyrenochaetaceae Valenz.-Lopez, Crous, Stchigel, Guarro \& Cano, Phaeosphaeriaceae M.E. Barr, Pleosporaceae Nitschke, Pseudopyrenochaetaceae Valenz.-Lopez, Crous, Stchigel, Guarro \& Cano, Pyrenochaetopsidaceae Valenz.-Lopez, Crous, Cano, Guarro \& Stchigel, Shiraiaceae Y.X. Liu, Zi Y. Liu \& K.D. Hyde and Tzeananiaceae Ariyaw., A.J.L. Phillips \& Chuang (Zhang et al. 2012, Hyde et al. 2013, Wanasinghe et al. 2017a, Ariyawansa et al. 2018). Many genera and families of Pleosporineae are well-resolved based upon their morphological characteristics coupled with phylogenetic affinities i.e. Camarosporidiellaceae, Cucurbitariaceae, Didymellaceae, Leptosphaeriaceae, Phaeosphaeriaceae and Pleosporaceae (Phookamsak et al. 2014, 2017, Ariyawansa et al. 2015a, b, Chen et al. 2017, Wanasinghe et al. 2017a, b, Jaklitsch et al. 2018). In contrast, some genera and families are not well-resolved. This is because of limited taxon sampling and brief protologues (Liu et al. 2016, 2017).

Barr (1989) accepted Dothidotthia as a member of Botryosphaeriaceae based on its morphological characters, such as immersed ascomata becoming erumpent to nearly superficial on woody twigs, broad and bitunicate asci. Nevertheless, multi-gene phylogenetic analyses showed that Dothidotthia resides in Pleosporales following which Phillips et al. (2008) introduced Dothidotthiaceae. Subsequent studies by Zhang et al. (2012), Hyde et al. (2013), Wijayawardene et al. (2014, 2018) and Liu et al. (2017) accepted this taxonomic arrangement. Species of Dothidotthiaceae have been commonly reported as parasitic on living plants, as well as saprobes on wood or branches of dead or decaying plants in terrestrial habitats (Hyde et al. 2013, Marin-Felix et al. 2017). Currently, Dothidotthia Höhn, Neodothidotthia Crous, Mycocentrospora Deighton, Phaeomycocentrospora Crous, H.D. Shin \& U. Braun, Pleiochaeta (Sacc.) S. Hughes, Thyrostroma Höhn. and Wilsonomyces Adask., J.M. Ogawa \& E.E. Butler are recognized in this family (MarinFelix et al. 2017, Wijayawardene et al. 2018, Crous et al. 2019). However, Mycocentrospora, Phaeomycocentrospora and Pleiochaeta exhibit different morphological characters from the type genus (especially in conidia shape and appendages) but were accommodated in Dothidotthiaceae.

The genus Thyrostroma was introduced by Höhnel (1911), with T. compactum (Sacc.) Höhn. as the type species. The generic synonym of Thyrostroma has been reported as Coryneum Sacc., Stegonsporium Corda, Stigmina Sacc., and Thyrococcum Höhn, Thyrostromella Höhnel and Wilsonomyces (Höhnel 1911, Morgan-Jones 1971, Sutton \& Pascoe 1989, Sutton 1997, Index Fungorum 2019). However, Thyrostromella is still treated in Ascomycota genera incertae sedis (Wijayawardene et al. 2018) and Coryneum, Stigmina, Stegonsporium and Wilsonomyces were accepted as distinct genera (Ramaley 2005, Marin-Felix et al. 2017, Wijayawardene et al. 2018). Thyrostroma species have been reported as plant pathogens causing stem canker and dieback on various hosts, with a cosmopolitan distribution (Yuan \& Old 1990, Kolemasova 1999, Kuz'michev et al. 2001, Sokolova 2003, Sokolova et al. 2006, Phillips et al. 2008, Kolganikhina \& Sokolova 2012, Stravinskienė et al. 2015, Crous et al. 2016, Marin-Felix et al. 2017, Farr \& Rossman 2019). However, based on morphology, Slippers et al. (2013) placed Thyrostroma in Botryosphaeriaceae. Dothidotthia has been reported as a sexual morph of Thyrostroma based on the production of hyphomycetes in culture from asci (Ramaley 2005). There is, however, no phylogenetic evidence for this link between these two genera. Wijayawardene et al. (2014) suggested that the usage of both names is desirable until the above-mentioned links are proven. Phylogenetic analyses based on LSU sequence data performed by Marin-Felix et al. (2017) showed that Thyrostroma clustered in 
the Dothidotthiaceae as originally proposed by Phillips et al. (2008). Furthermore, the type species of Thyrostroma, T. compactum formed a distinct clade from Dothidotthia in Dothidotthiaceae. It was therefore assumed that Thyrostroma and Dothidotthia are not congeneric (Marin-Felix et al. 2017). Although Thyrostroma is an old genus of Dothideomycetes (Wijayawardene et al. 2014), the taxonomic concepts and phylogenetic analyses of this genus remain unclear.

The objective of the present study was to clarify the taxonomic placement of Dothidotthia and Thyrostroma in Dothidotthiaceae and identify taxa based on morphology coupled with multigene phylogeny. Thirty-one thyrostroma-like taxa collected from Russia were examined and their DNA sequence data were obtained for use in multi-gene phylogenetic analyses.

\section{Materials \& Methods}

\section{Collections, isolation and identification}

Symptomatic specimens (Fig. 1) were collected from many hosts in different Provinces of Russia during 2015-2016. Collected specimens were brought to the laboratory in small paper bags. Specimens were examined with a Motic SMZ 168 series and the appearance of fruiting structures on the host surface was captured using a ZEISS STEREO Discovery.V8 stereomicroscope fitted with AxioCamERc5s camera. Squash mounts of fungal microscopic structures and free-hand sections of ascomata and sporodochia on glass slides in double-distilled water $\left(\mathrm{ddH}_{2} \mathrm{O}\right)$ were prepared for photography. Micro characteristics were examined and captured using a Nikon ECLIPSE 80i compound microscope, connected with a Cannon 600D digital camera and illustrated with DIC microscopy. Fungal structures were measured using Tarosoft ${ }^{\circledR}$ Image Framework program v.0.9.0.7. Photographic plates were made using Adobe Photoshop CS6 version 13.0.

Pure cultures were obtained by single spore isolation with spore suspension technique. The spore suspension was prepared by aseptically removing ascospores/conidia from the ascostroma/sporodochium and soaking them in a drop of sterilized water. The spore suspension was dropped on the surface of malt extract agar (MEA; $33.6 \mathrm{~g} / \mathrm{l}$ sterile distilled water, Difco malt extract media) and the droplets were spread over the agar surface with a sterile spreader. The MEA agar plate containing a spore suspension was incubated at $25-30^{\circ} \mathrm{C}$ for $4-24$ hours. Germinating ascospores/conidia were checked under the Motic SMZ 168 stereomicroscope after 4 hours and thereafter at 12-hour intervals and images captured with a Canon 600D camera on a Nikon ECLIPSE 80i microscope. Germinated ascospores/conidia were aseptically transferred to fresh MEA plates and colony diameters and culture characteristics were recorded after 1-4 weeks of incubation at $25-30^{\circ} \mathrm{C}$. Cultures were stored in screw cap tubes at $4^{\circ} \mathrm{C}$ and $-20^{\circ} \mathrm{C}$ for molecular work and incorporation into the culture collection. Herbarium specimens were deposited in the Mae Fah Luang University Herbarium, Chiang Rai, Thailand (MFLU). Cultures were deposited in Mae Fah Luang University Culture Collection (MFLUCC). The new species were justified based on the guidelines of Jeewon \& Hyde (2016). Faces of Fungi and Index Fungorum numbers were obtained based on the guidelines described in Jayasiri et al. (2015) and Index Fungorum (2019).

\section{DNA extraction, PCR amplification and DNA sequencing}

Cultures were grown on MEA at $25-30^{\circ} \mathrm{C}$ for two weeks and mycelia were scraped off and kept in $1.5 \mathrm{ml}$ sterilized tubes. Genomic DNA was extracted by Biospin Fungus Genomic DNA Extraction Kit (BioFlux ${ }^{\circledR}$, Hangzhou, P. R. China) following the manufacturer's protocol. In addition, DNA extraction of some samples was made directly from dry fungal fruiting bodies to obtain sequence data. Extraction was started by placing individual sporodochia in $1.5 \mathrm{ml}$ sterilized tubes and leaving overnight at $-20^{\circ} \mathrm{C}$. Genomic DNA was extracted by using OMEGA E.Z.N.A. ${ }^{\circledR}$ Forensic DNA Kit following the manufacturer's instructions. Polymerase chain reaction (PCR) was used to amplify partial gene regions (ITS, LSU, SSU, TEF1- $\alpha$, RPB2 and TUB2) using primers and conditions as shown in Table 1 . The amplification reactions were performed in $25 \mu 1$ final volumes contained $8.5 \mu \mathrm{l}$ of sterilized water, $12.5 \mu \mathrm{l}$ of $2 \times$ Easy Taq PCR SuperMix (a mixture of Easy Taq TM DNA Polymerase, dNTPs, and optimized buffer (Beijing Trans Gen Biotech Co., Chaoyang 
District, Beijing, PR China), $1 \mu 1$ of each forward and reverse primer (10 pM), and $2 \mu 1$ of DNA template. PCR fragments were purified and sequenced by Sangon Biotech Co., Shanghai, China and Sino Geno Max, Beijing, China.

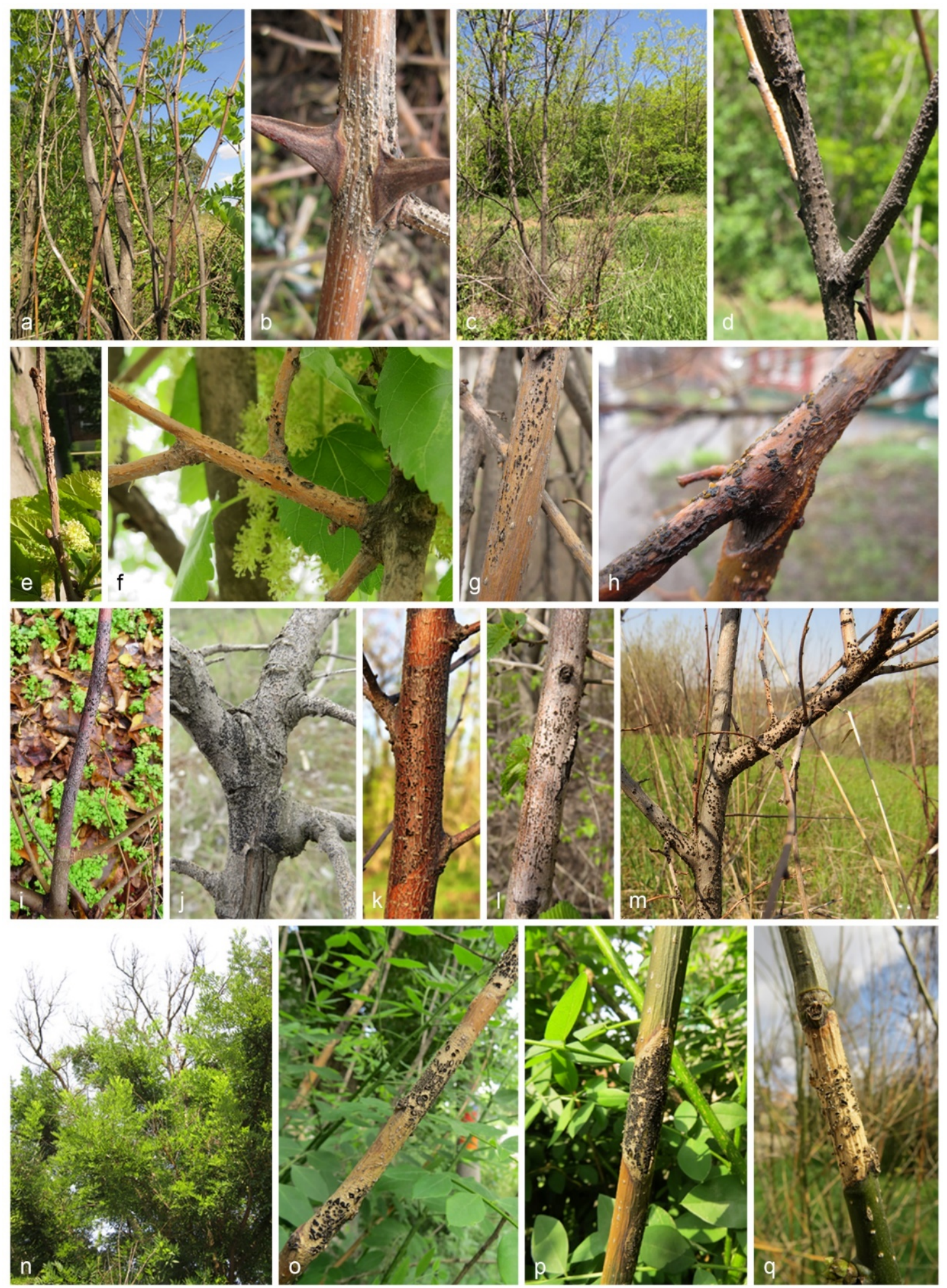

Figure 1 - Symptoms on different host caused by species of Dothidotthia and Thyrostroma. a, b Robinia pseudoacacia (D. robiniae). c, d Robinia pseudoacacia (T. robiniae). e-h Morus alba (T. moricola). i Tilia cordata (T. tiliae). j Ulmus pumila (T. tiliae). k, l Ulmus pumila (T. ulmicola). m Ulmus pumila (T. ulmigenum). n-q Styphnolobium japonicum (T. styphnolobii). 
Table 1 Details of genes/loci, primers used and PCR thermal cycles

\begin{tabular}{|c|c|c|c|}
\hline Gene/loci $^{a}$ & $\begin{array}{c}\text { PCR primers } \\
\text { (forward/reverse) }\end{array}$ & PCR conditions & References \\
\hline ITS & ITS5/ITS4 & 1. Initialization $=94^{\circ} \mathrm{C}$ for 3 minutes & White et al. 1990 \\
\hline LSU & LR0R/ LR5 & $\begin{array}{l}\text { 2. Denaturation }=94^{\circ} \mathrm{C}(1 \text { minute }) \\
\text { 3. Annealing }=55^{\circ} \mathrm{C}(50 \text { seconds })\end{array}$ & $\begin{array}{l}\text { Vilgalys \& Hester } \\
1990\end{array}$ \\
\hline SSU & NS1/NS4 & $\begin{array}{l}\text { 4. Extension }=72^{\circ} \mathrm{C}(1 \text { minute }) \\
\text { 5. Final elongation }=72^{\circ} \mathrm{C}(10 \text { minutes }) \\
\text { 6. Final hold at } 4^{\circ} \mathrm{C} \\
\text { (Step } 2-4=35 \text { cycles })\end{array}$ & White et al. 1990 \\
\hline TEF1- $\alpha$ & $\begin{array}{l}\text { EF1-983F/EF1- } \\
\text { 2218R }\end{array}$ & $\begin{array}{l}\text { 1. Initialization }=95^{\circ} \mathrm{C} \text { for } 3 \text { minutes } \\
\text { 2. Denaturation }=94^{\circ} \mathrm{C}(2 \text { minutes }) \\
\text { 3. Annealing }=56^{\circ} \mathrm{C}(1 \text { minute }) \\
\text { 4. Extension }=72^{\circ} \mathrm{C}(1 \text { minute }) \\
\text { 5. Final elongation }=72^{\circ} \mathrm{C}(10 \text { minutes }) \\
\text { 6. Final hold at } 4^{\circ} \mathrm{C} \\
\text { (Step } 2-4=35 \text { cycles })\end{array}$ & Rehner 2001 \\
\hline RPB2 & $\begin{array}{l}\text { fRPB2-5F/fRPB2- } \\
\text { 7cR }\end{array}$ & $\begin{array}{l}\text { 1. Initialization }=95^{\circ} \mathrm{C} \text { for } 5 \text { minutes } \\
\text { 2. Denaturation }=95^{\circ} \mathrm{C}(1 \text { minute }) \\
\text { 3. Annealing }=52^{\circ} \mathrm{C}(2 \text { minutes }) \\
\text { 4. Extension }=72^{\circ} \mathrm{C}(90 \text { seconds }) \\
\text { 5. Final elongation }=72^{\circ} \mathrm{C}(10 \text { minutes }) \\
\text { 6. Final hold at } 4^{\circ} \mathrm{C} \\
\text { (Step } 2-4=40 \text { cycles })\end{array}$ & Liu et al. 1999 \\
\hline TUB2 & $\mathrm{Bt} 2 \mathrm{a} / \mathrm{Bt} 2 \mathrm{~b}$ & $\begin{array}{l}\text { 1. Initialization }=94^{\circ} \mathrm{C} \text { for } 5 \text { minutes } \\
\text { 2. Denaturation }=94^{\circ} \mathrm{C}(1 \text { minute }) \\
\text { 3. Annealing }=58^{\circ} \mathrm{C}(30 \text { seconds }) \\
\text { 4. Extension }=72^{\circ} \mathrm{C}(90 \text { seconds }) \\
\text { 5. Final elongation }=72^{\circ} \mathrm{C}(10 \text { minutes }) \\
\text { 6. Final hold at } 4^{\circ} \mathrm{C} \\
\text { (Step } 2-4=37 \text { cycles })\end{array}$ & $\begin{array}{l}\text { Glass \& Donaldson } \\
1995\end{array}$ \\
\hline
\end{tabular}

${ }^{a}$ ITS: Part of rDNA 18S (3' end), the first internal transcribed spacer (ITS1), the 5.8S rRNA gene, the second ITS region (ITS2), and part of the 28S rRNA (5' end); LSU: 28S large subunit rDNA; SSU: 18S small subunit rDNA; TEF1- $\alpha$ : translation elongation factor 1-alpha gene; RPB2: RNA polymerase II second largest subunit; TUB2: $\beta$-tubulin

\section{Phylogenetic analyses}

The new LSU and ITS sequences generated in this study were subjected to BLASTn searches of the NCBI nucleotide database (http://blast.ncbi.nlm.nih.gov/) to determine their most probable closely related taxa. The sequences of representative taxa in Pleosporineae used in our phylogenetic analyses were selected from GenBank based on the BLASTn searches and recently published data (Marin-Felix et al. 2017, Wanasinghe et al. 2017a, Valenzuela-Lopez et al. 2018). Phylogenetic analyses in this study are represented by two phylogenetic trees. The first tree was generated from a combined LSU, SSU, ITS and TEF1- $\alpha$ gene dataset. The dataset comprises 131 sequence strains from 21 representative families in Pleosporineae, including the new taxa proposed in this study. Cyclothyriella rubronotata (CBS 121892 and CBS 141486) was selected as the outgroup taxon (Table 2).

The second phylogenetic tree was obtained from a combined ITS, LSU, SSU and TEF1- $\alpha$ gene dataset. The dataset consists of 55 sequence strains from six genera within Dothidotthiaceae. Didymella exigua (CBS 183.55) and Phoma herbarum (CBS 615.75) were selected as the outgroup taxa (Tables 2, 3). The individual gene alignments were initially aligned by MAFFT version 7 (Katoh et al. 2017; http://mafft.cbrc.jp/alignment/server/) and improved manually where necessary in BioEdit v.7.0.9.1 (Hall 1999) and MEGA7 (Kumar et al. 2015). The final alignments of the 
combined multi-gene dataset were analyzed and inferred the phylogenetic trees based on maximum likelihood (ML), maximum parsimony (MP) and Bayesian inference (BI) analyses.

Maximum likelihood )ML( analyses of the Pleosporineae and Dothidotthiaceae were performed by using the RAxML-HPC2 on XSEDE (v. 8.2.8) (Stamatakis et al. 2008, Stamatakis 2014) via the CIPRES Science Gateway platform (Miller et al. 2010) with the GTR $+\mathrm{I}+\mathrm{G}$ model of nucleotide substitution.

Maximum Parsimony (MP) analysis was carried out with PAUP v 4.0b10 (Swofford 2002). Trees were inferred using the heuristic search function with 1,000 random stepwise addition replicates and tree bisection-reconnection (TBR) as the branch-swapping algorithm. All informative characters were unordered and of equal weight. The consistency index (CI), retention index (RI), rescaled consistency index (RC) and homoplasy index (HI) were calculated. KishinoHasegawa tests (KHT) (Kishino \& Hasegawa 1989) were performed in order to determine whether trees were significantly different. Statistical supports for branches of the most parsimonious tree were estimated using maximum parsimony bootstrap (BS) analysis with 1,000 bootstrap replicates (Felsenstein 1985).

Bayesian inference (BI) analysis was performed by MrBayes on XSEDE, MrBayes 3.2.6 (Huelsenbeck \& Ronquist 2001) via the CIPRES Science Gateway platform (Miller et al. 2010). Bayesian posterior probabilities (BYPP) (Rannala \& Yang 1996, Zhaxybayeva \& Gogarten 2002) were evaluated by Markov Chain Monte Carlo sampling (BMCMC). Two parallel runs were conducted using the default settings, but with the following adjustments: Six simultaneous Markov chains were set up at 7,000,000 generations for Pleosporineae (Analyses 1) and 2,000,000 generations for Dothidotthiaceae (Analyses 2). Trees were sampled every $100^{\text {th }}$ generation. The distribution of log-likelihood scores was examined to determine the stationary phase for each search and to decide if extra runs were required to achieve convergence, using Tracer v. 1.6 program (Rambaut et al. 2013). The first $10 \%$ of generated trees representing the burn-in phase were discarded and the remaining trees were used to calculate posterior probabilities of the majority rule consensus tree.

The phylogenetic tree was visualized in FigTree v.1.4.3 (Rambaut 2016) and edited in Adobe Photoshop CS6 version 13.0. (Adobe Systems. U.S.A.). The newly generated sequences in this study were deposited in GenBank (Table 3). The final alignment and tree were deposited in TreeBASE (http://www.treebase.org/) under the submission ID. 24625, 25013 and 25016 (The analysis results of Pleosporineae of ML, MP and BI, respectively) and ID. 24626, 25019 and 25017 (The analysis results of Dothidotthiaceae of ML, MP and BI, respectively).

\section{Results}

\section{Phylogenetic analyses}

\section{Analysis for familial placement in Pleosporineae (Analyses 1)}

Phylogenetic analyses of the Pleosporineae based on the concatenated LSU, SSU, ITS and TEF1- $\alpha$ sequence dataset comprised 131 taxa and including 3197 bp (LSU: 1-808; SSU: 8091731; ITS: 1732-2332; TEF1- $\alpha$ : 2333-3197). The RAxML analysis of the combined gene dataset resulted in the best scoring likelihood tree selected with a final ML optimization likelihood value of -21537.730459 which is represented in Fig. 2. The dataset comprised 992 distinct alignment patterns, with $31.42 \%$ of undetermined characters or gaps. The MP analysis results: 2366 characters were constant, 84 variable characters were parsimony-uninformative, and 747 were (included) parsimony-informative characters. The most parsimonious tree is shown where $\mathrm{TL}=3529$, $\mathrm{CI}=$ $0.364, \mathrm{RI}=0.788, \mathrm{RC}=0.287, \mathrm{HI}=0.636$. Bayesian posterior probabilities from MCMC were evaluated with a final average standard deviation of split frequency $=0.009530$. It is noteworthy that we received a low consistency index (CI) for this character dataset in the MP analysis. We believe that the introns of ITS1 and ITS2 may provide a homoplasious nature to the dataset. Similar phylogenetic scenarios are reported with the inclusion of ITS in order/ suborder level, resulted in 
low CI values (Wanasinghe et al. 2017a, 2018). Phylogenies concerining to the placement of families in Pleosporineae were largely similar to those of Wanasinghe et al. (2017a), Ariyawansa et al. (2018) and Valenzuela-Lopez et al. (2018). However, some taxa were resolved differently in the internal node relationships among BI, ML and MP trees.

Twenty-five sequenced strains of Dothidotthia and Thyrostroma generated in this study are included in the analysis. Phylogenetic relationships of these taxa in Dothidotthiaceae and their relationships with other families in Pleosporineae are shown in Fig. 2. Phylogenetic results of this study show that Dothidotthiaceae forms a well-supported clade in Pleosporineae (100\% ML, 99\% MP, 1.00 PP). Families in Pleosporineae are well-resolved, except Neophaeosphaeriaceae which clusters with Neopyrenochaetaceae. Genera in Dothidotthiaceae form clearly distinct subclades and is confirmed by analyses 2 (Fig. 3). Dothidotthia is distinct from Thyrostroma and this corroborates previous studies (Crous et al. 2016, Marin-Felix et al. 2017) suggesting that they are not congeneric.

\section{Analysis for genera in Dothidotthiaceae (Analyses 2)}

Phylogenetic analyses of the Dothidotthiaceae based on the concatenated LSU, SSU, ITS and TEF1- $\alpha$ sequence data of 55 taxa included 3102 bp [LSU: 1-806; SSU: 807-1729; ITS: 17302236; TEF1- $\alpha$ : 2237-3102]. The RAxML analysis of the combined gene dataset had 295 distinct alignment patterns with $29.90 \%$ of undetermined characters or gaps. The best scoring of RAxML analysis is shown in Fig. 3, with the final ML optimization likelihood value of -6956.613576. In the MP analysis: 2827 characters were constant, 59 variable characters were parsimony-uninformative, and 216 were (included) parsimony-informative characters. The most parsimonious tree is shown where $\mathrm{TL}=453, \mathrm{CI}=0.682, \mathrm{RI}=0.902, \mathrm{RC}=0.615, \mathrm{HI}=0.318$. Bayesian posterior probabilities were evaluated by MCMC with a final average standard deviation of split frequencies $=0.007819$. ML, MP and BI analyses of a combined multi-gene phylogeny were similar in the overall tree topologies (data not shown) and concurred with Marin-Felix et al. (2017) and Crous et al. (2019).

To discuss phylogenetic analyses of taxa in Dothidotthiaceae (Fig. 3), we divided the ingroup taxa in Dothidotthiaceae into six subclades (A-F). Newly generated sequences from 19 isolates of thyrostroma-like taxa grouped with Thyrostroma compactum, T. cornicola, T. ephedricola, T. fransiriae and $T$. jaczewskii. These taxa formed a separate subclade (clade A) from other genera in Dothidotthiaceae, with moderate support in ML analysis and well-supported in MP and BI analyses (69\% ML, 95\% MP, 1.00 PP, Fig. 3). Eight Thyrostroma species viz. T. celtidis, T. lycii, T. moricola, T. robiniae, T. styphnolobii, T. tiliae, T. ulmicola and T. ulmigenum are introduced to accommodate these 19 isolates.

Four newly generated strains of a new species Dothidotthia robiniae (MFLUCC 16-1175, MFLUCC 16-1177, MFLUCC 16-1185, MFLUCC 18-0692) and two strains of D. negundinicola (MFLUCC 16-1157, MFLUCC 16-1183) grouped with D. negundinis (CPC 12928, CPC 12930, CPC 12932, CPC 12933), D. negundinicola (CBS145039) and D. symphoricarpi (CPC12929; type species). These taxa form a well-resolved clade in Dothidotthiaceae (Fig. 3; clade E). Dothidotthia has a close relationship with Mycocentrospora, Wilsonomyces and Pleiochaeta in analyses 1, however, the genus formed a distinct clade in analyses 2 (Fig. 3).

Wilsonomyces (clade B) is sister to Mycocentrospora (clade C) in analyses 2 (Fig. 3; clade B and C). Pleiochaeta (Fig. 3; clade D) forms a sister clade with Wilsonomyces and Mycocentrospora with significant support (90\% ML, 76\% MP, 1.00 PP, Fig. 3; clades B, C and D). Phaeomycocentrospora (Fig. 3; clade F) is represented by a putative species P. cantuariensis (E.S. Salmon \& Wormald) Crous, H.D. Shin \& U. Braun, the genus forms a well-resolved clade basal to Dothidotthiaceae (100\% ML, 100\% MP, 1.00 PP). 
Table 2 GenBank and culture collection accession numbers of taxa in Pleosporineae used in this study.

\begin{tabular}{|c|c|c|c|c|c|}
\hline \multirow{2}{*}{ Taxon } & \multirow{2}{*}{ Culture no. } & \multicolumn{4}{|c|}{ GenBank accession number } \\
\hline & & LSU & SSU & ITS & TEF1- $\alpha$ \\
\hline Acrocalymma aquatica & MFLUCC 11-0208 & JX276952 & JX276953 & NR_121544 & - \\
\hline Acrocalymma ficus & CBS $317.76^{\mathrm{T}}$ & KP170712 & - & NR_137953 & KP170663 \\
\hline Acrocalymma medicaginis & CPC $24340^{\mathrm{T}}$ & KP170718 & - & KP170625 & - \\
\hline Acrocalymma medicaginis & СРC 24345 & KP170713 & - & KP170620 & - \\
\hline Alternaria alternata & MFLUCC 14-1184 & KP334701 & КР334721 & KP334711 & KP334735 \\
\hline Alternaria eureka & CBS $193.86^{\mathrm{T}}$ & KC584331 & KC584589 & - & - \\
\hline Alternariaster helianthi & CBS $327.69^{\mathrm{T}}$ & KC584369 & KC584627 & KC609335 & - \\
\hline Ascochyta pisi & CBS $126.54^{\mathrm{T}}$ & DQ678070 & DQ678018 & - & DQ677913 \\
\hline Ascocylindrica marina & MD6011 & KT252905 & KT252907 & - & - \\
\hline Ascocylindrica marina & MD6012 & KT252906 & - & - & - \\
\hline Boeremia exigua var. exigua & CBS $431.74^{\mathrm{T}}$ & EU754183 & EU754084 & FJ427001 & GU349080 \\
\hline Camarosporidiella caraganicola & MFLUCC $14-0605^{\mathrm{T}}$ & KP711381 & KP711382 & KP711380 & - \\
\hline Camarosporidiella celtidis & MFLUCC 14-0904 & MF434217 & MF434305 & MF434129 & MF434392 \\
\hline Camarosporidiella elaeagnicola & MFLUCC 14-0908 & MF434225 & MF434313 & MF434137 & MF434400 \\
\hline Camarosporidiella halimodendri & MFLUCC $14-0901^{\mathrm{T}}$ & MF434234 & MF434322 & MF434146 & MF434409 \\
\hline Camarosporidiella laburni & MFLUCC 14-0885 & MF434241 & MF434329 & MF434153 & MF434416 \\
\hline Camarosporidiella schulzeri & MFLUCC $14-0897^{\mathrm{T}}$ & MF434273 & MF434361 & MF434185 & MF434448 \\
\hline Camarosporium quaternatum & CPC 23216 & KY929170 & KY929122 & KY929135 & KY929200 \\
\hline Camarosporium quaternatum & CPC $31081^{\mathrm{T}}$ & KY929171 & KY929123 & KY929136 & KY929201 \\
\hline Camarosporium quaternatum & CPC 31518 & KY929172 & KY929124 & KY929137 & KY929202 \\
\hline Cucurbitaria berberidis & CBS 363.93 & GQ387606 & GQ387545 & JF740191 & - \\
\hline Cucurbitaria berberidis & MFLUCC 11-0386 & KC506795 & KC506799 & - & - \\
\hline Cucurbitaria ephedricola & $\mathrm{HA} 42^{\mathrm{T}}$ & КТ313007 & KT313005 & - & - \\
\hline Cyclothyriella rubronotata & CBS 121892 & - & - & KX650541 & KX650516 \\
\hline Cyclothyriella rubronotata & CBS $141486^{\mathrm{T}}$ & KX650544 & KX650507 & KX650544 & KX650519 \\
\hline Didymella exigua & CBS $183.55^{\mathrm{T}}$ & EU754155 & EU754056 & GU237794 & - \\
\hline Dothidotthia negundinis & СРC 12928 & EU673272 & EU673225 & MK442598 & - \\
\hline Dothidotthia negundinis & CPC 12930 & EU673274 & EU673226 & MK442599 & - \\
\hline Dothidotthia negundinis & CPC 12932 & EU673275 & EU673227 & MK442600 & - \\
\hline Dothidotthia negundinis & СРC 12933 & EU673276 & EU673228 & MK442601 & - \\
\hline Dothidotthia symphoricarpi & CPC $12929^{\mathrm{T}}$ & EU673273 & EU673224 & - & - \\
\hline Dothidotthia symphoricarpi & CBS $119687^{\mathrm{T}}$ & MH874618 & - & MH863064 & - \\
\hline Dothidotthia negundinicola & CBS $145039^{\mathrm{T}}$ & MK442537 & - & MK442597 & - \\
\hline Foliophoma fallens & CBS 161.78 & GU238074 & GU238215 & KY929147 & - \\
\hline Foliophoma fallens & CBS 284.70 & GU238078 & GU238218 & KY929148 & - \\
\hline Halojulella avicenniae & BCC 18422 & GU371822 & GU371830 & - & GU371815 \\
\hline Halojulella avicenniae & BCC 20173 & GU371823 & GU371831 & - & GU371816 \\
\hline
\end{tabular}


Table 2 Continued.

\begin{tabular}{|c|c|c|c|c|c|}
\hline \multirow{2}{*}{ Taxon } & \multirow{2}{*}{ Culture no. } & \multicolumn{4}{|c|}{ GenBank accession number } \\
\hline & & LSU & SSU & ITS & TEF1- $\alpha$ \\
\hline Halojulella avicenniae & JK5326A & GU479790 & GU479756 & - & - \\
\hline Leptosphaeria maculans & CBS 260.94 & JF740307 & - & JF740235 & - \\
\hline Leptosphaerulina australis & CBS 317.83 & GU301830 & GU296160 & GU237829 & GU349070 \\
\hline Libertasomyces myopori & CPC $27354^{\mathrm{T}}$ & KX228332 & - & NR_145200 & - \\
\hline Libertasomyces platani & CPC $29609^{\mathrm{T}}$ & KY173507 & - & KY173416 & - \\
\hline Libertasomyces quercus & CBS $134.97^{\mathrm{T}}$ & DQ377883 & - & KY929152 & KYY929197 \\
\hline Microsphaeropsis olivacea & CBS 233.77 & N712563 & - & JN712497 & - \\
\hline Microsphaeropsis proteae & CBS 111319 & GU237988 & - & GU237803 & - \\
\hline Mycocentrospora acerina & CBS 148.52 & MH868490 & - & MH856968 & - \\
\hline Mycocentrospora acerina & CBS 113.24 & MH866268 & - & MH854764 & - \\
\hline Neocamarosporium lamiacearum & MFLUCC $17-0560^{\mathrm{T}}$ & MF434279 & MF434367 & MF434191 & MF434454 \\
\hline Neocamarosporium lamiacearum & MFLUCC 17-0750 & MF434280 & MF434368 & MF434192 & MF434455 \\
\hline Neocamarosporium salicorniicola & MFLUCC 15-0957 & MF434281 & MF434369 & MF434192 & - \\
\hline Neocamarosporium salsolae & MFLUCC $17-0827^{\mathrm{T}}$ & MF434283 & MF434371 & MF434195 & MF434457 \\
\hline Neophaeosphaeria agaves & CPC $21264^{\mathrm{T}}$ & KF777227 & - & KF777174 & - \\
\hline Neophaeosphaeria filamentosa & CBS 102202 & GQ387577 & GQ387516 & JF740259 & $\bar{G} U 349084$ \\
\hline Neopyrenochaeta acicola & CBS $812.95^{\mathrm{T}}$ & GQ387602 & GQ387541 & LT623218 & - \\
\hline Neopyrenochaeta fragariae & CBS $101634^{\mathrm{T}}$ & GQ387603 & GQ387542 & LT623217 & - \\
\hline Paraleptosphaeria dryadis & CBS $643.86^{\mathrm{T}}$ & KC584632 & GU301828 & JF740213 & GU349009 \\
\hline Paraleptosphaeria rubi & MFLUCC $14-0211^{\mathrm{T}}$ & KT454718 & KT454733 & KT454726 & - \\
\hline Parapyrenochaeta acaciae & CBS $141291^{\mathrm{T}}$ & KX228316 & - & KX228265 & - \\
\hline Parapyrenochaeta protearum & CBS $131315^{\mathrm{T}}$ & JQ044453 & - & JQ044434 & - \\
\hline Parapyrenochaeta protearum & CBS 137997 & KJ869209 & - & KJ869152 & - \\
\hline Phaeomycocentrospora cantuariensis & СРC 10157 & GU253712 & - & GU269664 & GU384381 \\
\hline Phaeomycocentrospora cantuariensis & СРC 10762 & GU253713 & - & GU269665 & GU384382 \\
\hline Phaeomycocentrospora cantuariensis & СРC 11646 & GU253715 & - & GU269667 & GU384384 \\
\hline Phaeomycocentrospora cantuariensis & CPC 11694 & GU253716 & - & GU269668 & - \\
\hline Phaeosphaeria chiangraina & MFLUCC $13-0231^{\mathrm{T}}$ & KM434280 & KM434289 & KM434270 & KM434298 \\
\hline Phaeosphaeria musae & MFLUCC 11-0133 & KM434277 & KM434287 & KM434267 & KM434296 \\
\hline Phaeosphaeria thysanolaenicola & MFLUCC 10-0563 & KM434276 & KM434286 & KM434266 & KM434295 \\
\hline Phaeosphaeriopsis dracaenicola & MFLUCC 11-0157 & KM434283 & KM434292 & KM434273 & KM434301 \\
\hline Phaeosphaeriopsis dracaenicola & MFLUCC 11-0193 & KM434284 & KM434293 & KM434274 & KM434302 \\
\hline Pleiochaeta carotae & CBS $142644^{\mathrm{T}}$ & KY905663 & - & KY905669 & - \\
\hline Pleiochaeta ghindensis & CBS $552.92^{\mathrm{T}}$ & - & - & EU167561 & - \\
\hline Pleiochaeta setosa & CBS 496.63 & - & - & EU167563 & - \\
\hline Pleiochaeta setosa & DB50112 & - & - & JQ358708 & - \\
\hline Pleiochaeta setosa & 487630 & - & - & KR536610 & - \\
\hline
\end{tabular}


Table 2 Continued.

\begin{tabular}{|c|c|c|c|c|c|}
\hline \multirow{2}{*}{ Taxon } & \multirow{2}{*}{ Culture no. } & \multicolumn{4}{|c|}{ GenBank accession number } \\
\hline & & LSU & SSU & ITS & TEF1- $\alpha$ \\
\hline Plenodomus guttulatus & MFLUCC 15-1876 & KT454713 & KT454729 & KT454721 & - \\
\hline Plenodomus salviae & MFLUCC 13-0219 & KT454717 & KT454732 & KT454725 & - \\
\hline Pleospora herbarum & CBS 191.86 & JX681120 & - & JX681120 & KC584471 \\
\hline Phoma herbarum & CBS 615.75 & KF251715 & EU754087 & KF251212 & KR184186 \\
\hline Pseudopyrenochaeta lycopersici & CBS $306.65^{\mathrm{T}}$ & EU754205 & EU754106 & NR_103581 & - \\
\hline Pseudopyrenochaeta terrestris & CBS $282.72^{\mathrm{T}}$ & LT623216 & - & LT623228 & - \\
\hline Pyrenochaetopsis confluens & CBS $142459^{\mathrm{T}}$ & LN907446 & - & LT592950 & - \\
\hline Pyrenochaetopsis decipiens & CBS $343.85^{\mathrm{T}}$ & GQ387624 & - & LT623223 & - \\
\hline Pyrenochaetopsis indica & CBS $124454^{\mathrm{T}}$ & GQ387626 & - & LT623224 & - \\
\hline Shiraia bambusicola & NBRC 30753 & AB354968 & - & AB354987 & - \\
\hline Shiraia bambusicola & NBRC 30754 & AB354969 & - & AB354988 & - \\
\hline Shiraia bambusicola & NBRC 30771 & AB354971 & - & AB354990 & - \\
\hline Shiraia bambusicola & NBRC 30772 & AB354972 & - & AB354991 & - \\
\hline Staurosphaeria aloes & CPC $21572^{\mathrm{T}}$ & KF777198 & - & KF777142 & - \\
\hline Staurosphaeria lycii & MFLUCC $17-0210^{\mathrm{T}}$ & MF434284 & MF434372 & MF434196 & MF434458 \\
\hline Staurosphaeria lycii & MFLUCC 17-0211 & MF434285 & MF434373 & MF434197 & MF434459 \\
\hline Staurosphaeria lycii & MFLUCC 17-0720 & MF434286 & MF434374 & MF434198 & MF434460 \\
\hline Staurosphaeria rhamnicola & MFLUCC 17-0814 & MF434289 & MF434377 & MF434201 & MF434463 \\
\hline Subplenodomus valerianae & CBS 630.68 & GU238150 & GU238229 & JF740251 & - \\
\hline Subplenodomus violicola & CBS 306.68 & GU238156 & GU238231 & FJ427083 & - \\
\hline Thyrostroma compactum & CBS 335.37 & KY905664 & - & KY905670 & KY905681 \\
\hline Thyrostroma cornicola & CBS $141280^{\mathrm{T}}$ & KX228300 & - & KX228248 & KX228372 \\
\hline Thyrostroma ephedricola & MFLUCC 18-1125 & MK765854 & MK765853 & MK765855 & - \\
\hline Thyrostroma franseriae & CBS $487.71^{\mathrm{T}}$ & KX228301 & - & KX228249 & KY905680 \\
\hline Thyrostroma franseriae & CBS 700.70 & KX228302 & - & KX228250 & KY905682 \\
\hline Thyrostroma jaczewskii & MFLUCC 18-0787 & MK765857 & MK765858 & MK765856 & - \\
\hline Tzeanania taiwanensis & NTUCC 17-005 & MH461120 & MH461126 & MH461123 & MH461130 \\
\hline Tzeanania taiwanensis & NTUCC 17-006 & MH461121 & MH461127 & MH461124 & MH461131 \\
\hline Wilsonomyces carpophilus & CBS 147.36 & KY905667 & - & - & - \\
\hline Wilsonomyces carpophilus & CBS 159.51 & KY905665 & - & KY905671 & KY905683 \\
\hline Wilsonomyces carpophilus & CBS 231.89 & KY905666 & - & KY905672 & - \\
\hline Wilsonomyces carpophilus & SF4 & - & - & MF055699 & - \\
\hline
\end{tabular}

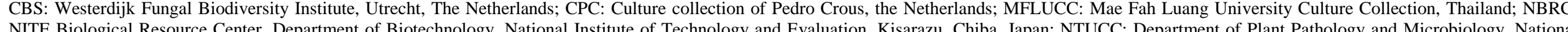
Taiwan University Culture Collection.

ITS: internal transcribed spacers and intervening 5.8S nrRNA; LSU: partial 28S large subunit RNA gene; SSU: partial 18S small subunit RNA gene; TEF1- $\alpha$ : partial translation elongation factor 1-alpha gene. The ex-type strains are noted with superscripted T. 
Table 3 GenBank and culture collection accession numbers of taxa in Dothidotthiaceae obtained in this study.

\begin{tabular}{|c|c|c|c|c|c|c|c|c|c|}
\hline \multirow[t]{2}{*}{ Taxon } & \multirow{2}{*}{$\begin{array}{l}\text { Original } \\
\text { no. }\end{array}$} & \multirow[t]{2}{*}{ Strain } & \multirow[t]{2}{*}{ Specimens no. } & \multicolumn{6}{|c|}{ GenBank accession number } \\
\hline & & & & LSU & SSU & ITS & TEF1- $\alpha$ & RPB2 & TUB2 \\
\hline \multirow{2}{*}{ Dothidotthia negundinicola } & $\mathrm{T}-1288$ & MFLUCC 16-1157 & MFLU 16-1582 & MK751815 & MK751760 & MK751725 & MK908015 & MK920235 & MK933784 \\
\hline & $\mathrm{T}-1465$ & MFLUCC 16-1183 & MFLU 16-1759 & MK751816 & MK751761 & MK751726 & MK908016 & MK920236 & MK933785 \\
\hline \multirow[t]{5}{*}{ Dothidotthia robiniae } & T-1369 & MFLUCC 16-1175 & MFLU 16-1663 & MK751817 & MK751762 & MK751727 & MK908017 & MK920237 & MK933786 \\
\hline & $\mathrm{T}-1410$ & MFLUCC 16-1177 & MFLU 16-1704 & MK751818 & MK751763 & MK751728 & MK908018 & - & MK933787 \\
\hline & $\mathrm{T}-1504$ & MFLUCC 16-1185 & MFLU 16-1798 & MK751819 & MK751764 & MK751729 & MK908019 & MK920238 & MK933788 \\
\hline & & MFLU 16-1798 & MFLU 16-1798 & MK751820 & MK751765 & MK751730 & MK908020 & MK920239 & MK933789 \\
\hline & T-1509 & MFLUCC 18-0692 & MFLU 16-1803 & MK751821 & MK751766 & MK751731 & MK908021 & MK920240 & MK933790 \\
\hline Thyrostroma celtidis & T-1506 & MFLUCC 16-1186 & MFLU 16-1800 & MK751822 & MK751767 & MK751732 & MK908022 & - & MK933791 \\
\hline Thyrostroma moricola & T-1501 & MFLU 16-1795 & MFLU 16-1795 & MK751823 & MK751768 & MK751733 & MK908023 & - & MK933792 \\
\hline Thyrostroma lycii & $\mathrm{T}-1348$ & MFLUCC 16-1170 & MFLU 16-1642 & MK751824 & MK751769 & MK751734 & MK908024 & MK920241 & MK933793 \\
\hline Thyrostroma robiniae & T-1504B & MFLUCC 18-1191 & MFLU 18-0631 & MK751825 & MK751770 & MK751735 & MK908025 & MK920242 & MK933794 \\
\hline Thyrostroma styphnolobii & T-1325 & MFLUCC 16-1160 & MFLU 16-1619 & MK751826 & MK751771 & MK751736 & MK908026 & MK920243 & MK933795 \\
\hline \multirow[t]{4}{*}{ Thyrostroma tiliae } & $\mathrm{T}-1387$ & MFLUCC 16-1176 & MFLU 16-1681 & MK751827 & MK751772 & MK751737 & MK908027 & MK920244 & MK933796 \\
\hline & T-1446 & MFLUCC 16-1178 & MFLU 16-1740 & MK751828 & MK751773 & MK751738 & MK908028 & MK920245 & MK933797 \\
\hline & $\mathrm{T}-1454 \mathrm{~B}$ & MFLUCC 16-1180 & MFLU 18-0628 & MK751829 & MK751774 & MK751739 & MK908029 & - & MK933798 \\
\hline & $\mathrm{T}-1519$ & MFLUCC 16-1188 & MFLU 16-1813 & MK751830 & MK751775 & MK751740 & MK908030 & MK920246 & MК933799 \\
\hline \multirow[t]{14}{*}{ Thyrostroma ulmicola } & T-1295 & MFLUCC 16-1158 & MFLU 16-1589 & MK751831 & MK751776 & MK751741 & MK908031 & MK920247 & MK933800 \\
\hline & T-1326 & MFLUCC 16-1161 & MFLU 16-1620 & MK751832 & MK751777 & MK751742 & MK908032 & MK920248 & MK933801 \\
\hline & $\mathrm{T}-1327$ & MFLUCC 16-1162 & MFLU 16-1621 & MK751833 & MK751778 & MK751743 & MK908033 & MK920249 & MK933802 \\
\hline & $\mathrm{T}-1328 \mathrm{~A}$ & MFLUCC 16-1163 & MFLU 16-1622 & MK751834 & MK751779 & MK751744 & MK908034 & MK920250 & MK933803 \\
\hline & $\mathrm{T}-1329$ & MFLUCC 16-1165 & MFLU 16-1623 & MK751835 & MK751780 & MK751745 & MK908035 & MK920251 & MK933804 \\
\hline & T-1330B & MFLUCC 16-1167 & MFLU 18-0627 & MK751836 & MK751781 & MK751746 & MK908036 & MK920252 & MK933805 \\
\hline & $\mathrm{T}-1331$ & MFLUCC 16-1168 & MFLU 16-1625 & MK751837 & MK751782 & MK751747 & MK908037 & MK920253 & MK933806 \\
\hline & & MFLUCC 16-1169 & MFLU 16-1625 & MK751838 & MK751783 & MK751748 & MK908038 & MK920254 & MK933807 \\
\hline & $\mathrm{T}-1357$ & MFLUCC 16-1711 & MFLU 16-1651 & MK751839 & MK751784 & MK751749 & MK908039 & MK920255 & MK933808 \\
\hline & T-1358 & MFLUCC 16-1172 & MFLU 16-1652 & MK751840 & MK751785 & MK751750 & MK908040 & MK920256 & - \\
\hline & & MFLUCC 16-1173* & MFLU 16-1652 & MK751841 & MK751786 & MK751751 & MK908041 & MK920257 & MK933809 \\
\hline & $\mathrm{T}-1454 \mathrm{~A}$ & MFLUCC 16-1179 & MFLU 16-1748 & MK751842 & MK751787 & MK751752 & MK908042 & MK920258 & MK933810 \\
\hline & $\mathrm{T}-1455$ & MFLUCC 16-1181 & MFLU 16-1749 & MK751843 & MK751788 & MK751753 & MK908043 & MK920259 & MK933811 \\
\hline & $\mathrm{T}-1459$ & MFLUCC 16-1182 & MFLU 16-1753 & MK751844 & MK751789 & MK751754 & MK908044 & MK920260 & MK933812 \\
\hline \multirow[t]{2}{*}{ Thyrostroma ulmigenum } & $\mathrm{T}-1328 \mathrm{~B}$ & MFLUCC 16-1164 & MFLU 18-0629 & MK751845 & MK751790 & MK751755 & MK908045 & MK920261 & МК933813 \\
\hline & T-1330A & MFLUCC 16-1166 & MFLU 16-1624 & MK751846 & MK751791 & MK751756 & MK908046 & MK920262 & MK933814 \\
\hline
\end{tabular}




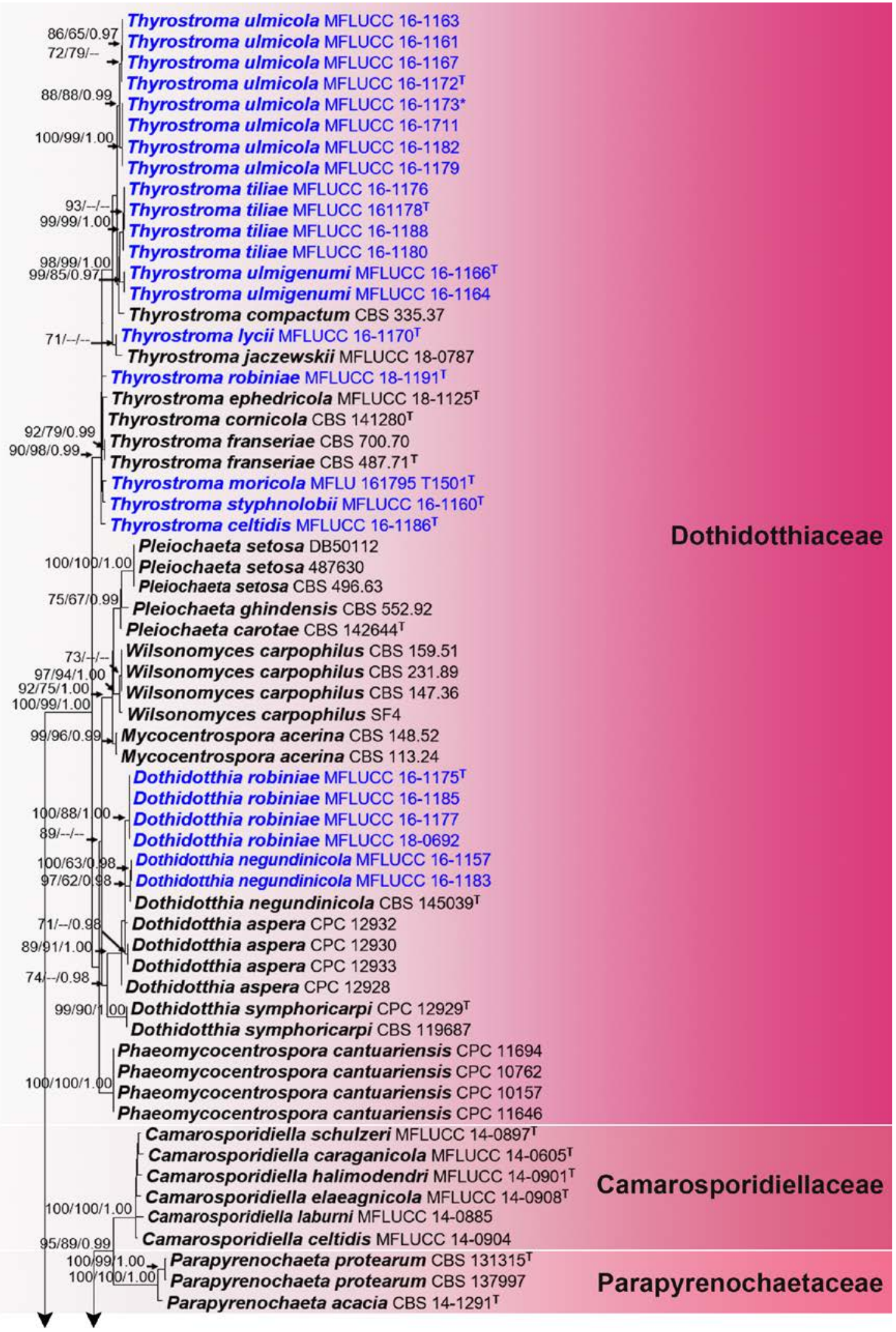

Figure 2 - Phylogram generated from RAxML analysis of the families in Pleosporineae based on a combined LSU, SSU, ITS and TEF1- $\alpha$ sequence dataset. Bootstrap support values for ML and MP equal to or greater than $60 \%$, and Bayesian posterior probabilities (PP) equal to or greater than 0.95 are defined as ML/MP/PP above the nodes. The tree is rooted to Cyclothyriella rubronotata (CBS 121892 and CBS 141486). The new isolates are in blue. Asterisk marks show the origin of the Dothidotthia and Thyrostroma isolates from ascospores. Ex-type strains are noted with superscript T. 




Figure 2 - Continued. 


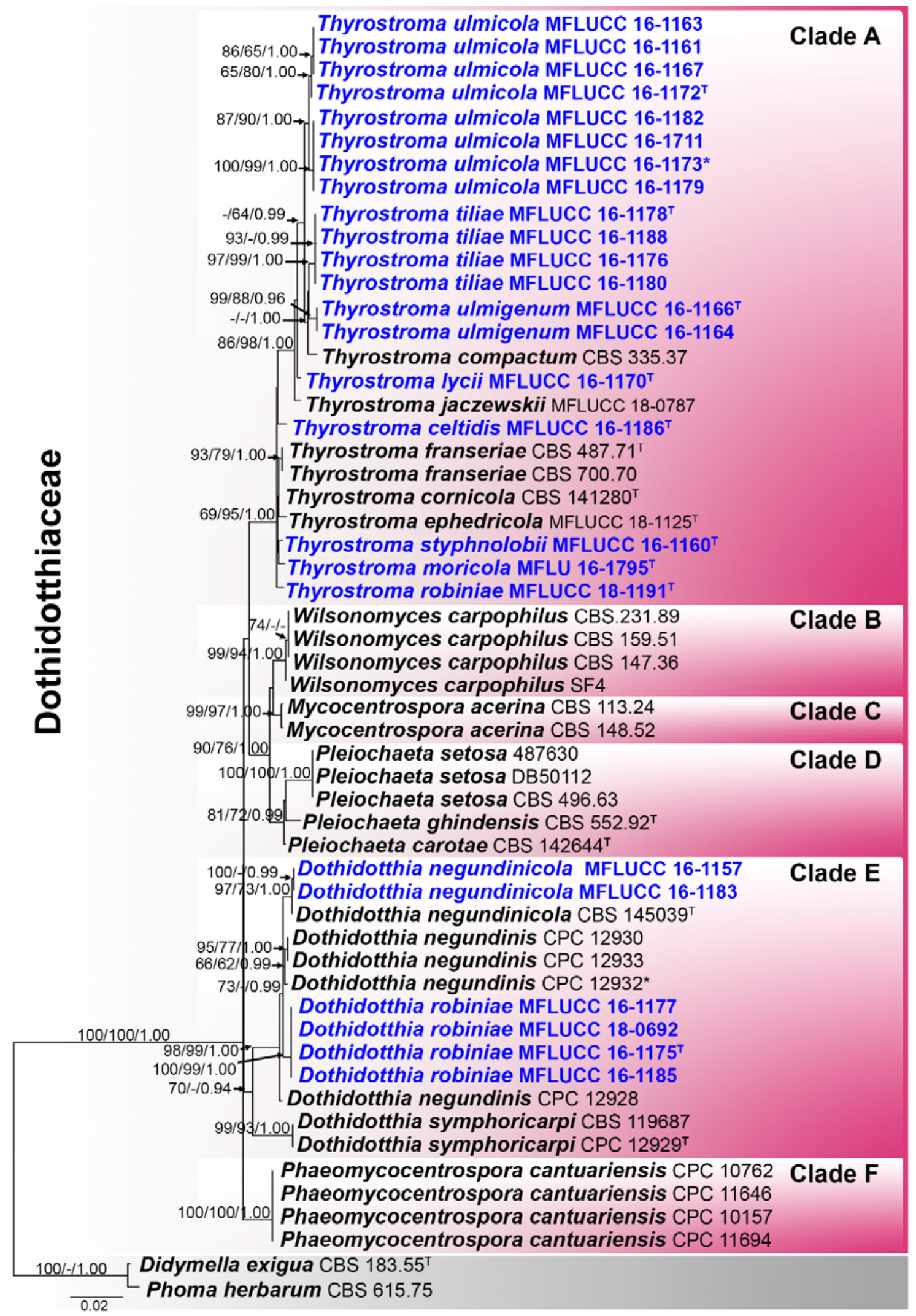

Figure 3 - Phylogram generated for Dothidotthiaceae from RAxML analysis based on a combined dataset of LSU, SSU, ITS and TEF1- $\alpha$ sequence dataset. Bootstrap support values for ML and MP equal to or greater than $60 \%$, and Bayesian posterior probabilities (PP) equal to or greater than 0.95 are defined as ML/MP/PP above the nodes. The tree is rooted to Didymella exigua (CBS 183.55) and Phoma herbarum (CBS 615.75). The new isolates are in blue. Asterisk marks show the origin of the Dothidotthia and Thyrostroma isolates from ascospores. Ex-type strains are noted with superscript $\mathrm{T}$. 


\section{Taxonomy}

Dothidotthiaceae Crous \& A.J.L. Phillips, Persoonia 21: 35 (2008)

Saprobic, pathogenic on leaves, branches, twigs and wood in terrestrial habitats. Sexual morph: Ascostromata solitary to gregarious, raised to erumpent, breaking through host surface, ruptured, rounded, elongate, lenticular, or irregular in shape, multi-loculate, glabrous, ostiolate. Ascomata coriaceous, globose to subglobose, dark brown to black, ostiolate with apex somewhat papillate to depress. Peridium consists of dark brown to black, scleroplectenchymatous cells of textura angularis to globulosa, or textura prismatica in between the locules, basal region giving rise, dark brown, thick-walled hyphae extending from the base of the ascostroma into the host substrate. Hamathecium composed of dense, broad, septate, branched, anastomosed pseudoparaphyses, embedded in a gelatinous matrix. Asci 8-spored, bitunicate, fissitunicate, clavate, with a short pedicel, rounded at the apex with an ocular chamber. Ascospores fusiform to ellipsoidal, pale to medium brown, 1-septate, slightly constricted at the septum, smooth-walled, thin-walled, with or without a gelatinous sheath. Asexual morph: Colonies punctiform, brown to black, stromatic, visible as raised, erumpent or nearly superficial, brown to dark brown, pulvinate sporodochia. Conidiophores macronematous, mononematous, short and packed, usually unbranched, septate or aseptate, straight or flexuous, hyaline to brown, or olivaceous brown, smooth or verrucose. Conidiogenous cells monoblastic, holoblatic, polyblastic, annelledic, integrated, terminal, or intercalary, or conidiophores reduced to conidiogenous cells, percurrent, cylindrical to subcylindrical. Conidia varied in shape, clavate to obclavate, cylindrical, ellipsoid or fusiform, filiform, subhyaline to dark brown, euseptate, phragmospores or muriform, smooth or rough, verrucose or echinulate, with or without appendages around the apical cell (Phillips et al. 2008, Hyde et al. 2013, Marin-Felix et al. 2017).

Type genus - Dothidotthia Höhn.

Notes - Barr (1989) included Dothidotthia in Botryosphaeriaceae with its coelomycetous asexual morph (as Dothiorella; fide Crous et al. 2006), and the characteristic of peridium, pseudoparaphyses and asci. However, Ramaley (2005) reported the asexual morph of Dothidotthia aspera as a hyphomycete in Thyrostroma. Considering the asexual morph differences together with molecular support, Phillips et al. (2008) established Dothidotthiaceae to accommodate Dothidotthia and proposed Thyrostroma as the asexual morph of Dothidotthia. Marin-Felix et al. (2017), however, showed that Dothidotthia is phylogenetically distinct from Thyrostroma and proposed that these two genera are not congeneric. Our phylogenetic results concur with Marin-Felix et al. (2017) and thus, we treat that Dothidotthia and Thyrostroma are two distinct genera.

Dothidotthia Höhn., Berichte der Deutschen Botanischen Gesellschaft 36: 312 (1918)

Saprobic or pathogenic on leaves and wood in terrestrial habitats (Fig. 1). Sexual morph: Ascostromata solitary to clustered, gregarious, raised to erumpent, breaking through host surface, rounded, elongate, lenticular or irregular in shape, multi-loculate, glabrous, ostiolate. Ascomata coriaceous, globose to subglobose, dark brown to black, ostiolate, apapillate. Peridium composed of 3-6 layers of dark brown to black cells of textura angularis, thick-walled, the basal region extending into the host substrate. Hamathecium composed of dense, broad, septate, branched pseudoparaphyses, anastomosing above the asci, embedded in a gelatinous matrix. Asci 8-spored, bitunicate, fissitunicate, clavate, short pedicellate, apically rounded with a well-developed ocular chamber. Ascospores ellipsoid, pale brown to medium brown, obtuse at ends or somewhat acute, transversely 1-septate, constricted at the septum (Phillips et al. 2008). Asexual morph: Colonies partly immersed, stromatic, effuse, sporodochial, with basal pseudoparenchymatous stroma, erumpent, dark brown to black. Conidiophores macronematous, septate, branched, subhyaline, smooth, arising from basal colonies. Conidiogenous cells enteroblastic, annellidic, integrated, terminal. Conidia acrogenous, fusiform to obclavate or obpyriform, pale brown to brown, rounded at apex, truncate at base, with a protruding hilum, septate, 0-3-transversely septate, constricted at the septa, rough-walled, echinulate. 
Type species - Dothidotthia symphoricarpi (Rehm) Höhn.

Notes - According to Ramaley (2005), Dothidotthia was the sexual morph of Thyrostroma based on a cultural examination, however, there is no further evidence to confirm this link. There are very few molecular data-based studies of Dothidotthia compared to morphological studies. It is problematic to confirm the link between sexual and asexual morphs of Dothidotthia only based on morphological characteristics. Currently, there are 11 epithets listed in this genus (Index Fungorum 2019) and 106 sequences (including our sequences in this study) are available in GenBank (accessed on $15^{\text {th }}$ August 2019). In this study, Dothidotthia forms a single clade, separate from Thyrostroma. Therefore, Dothidotthia and Thyrostroma are considered as distinct genera based on the sexual and asexual morph differences coupled with phylogenetic support.

Neodothidotthia Crous was introduced by Crous et al. (2019) with N. negundinicola Crous \& Akulov as the type species. The genus was introduced to accommodate $N$. negundinicola and $N$. negundinis (Berk. \& M.A. Curtis) Crous (三 Coryneum negundinis Berk. \& M.A. Curtis) based only on LSU sequences. In this study (based on a concatenated LSU, SSU, ITS and TEF1- $\alpha$ sequence dataset), Neodothidotthia forms a well-resolved clade in Dothidotthia (73\% ML, 0.99 PP, Fig. 3). We could not find any significant morphological differences between these two genera and based on the phylogenetic results obtained herein, it is wise to consider both of them as one genus. We, therefore, synonymize Neodothidotthia under Dothidotthia. Thus, a novel species Dothidotthia robiniae and two new combinations, $D$. negundinicola and $D$. negundinis are proposed.

Dothidotthia negundinicola (Crous \& Akulov) Senwanna, Wanas., Bulgakov, Phookamsak \& K.D. Hyde, comb. nov.

Index Fungorum number: IF556640; Facesoffungi number: FoF06139

Fig. 4

Basionym: Neodothidotthia negundinicola Crous \& Akulov, in Crous et al., Fungal Systematics and Evolution 3: 93 (2019)

Holotype - UKRAINE, Kharkiv region, Zolochiv District, on dead aerially attached branches of Acer negundo (Sapindaceae), 28 May 2017, A. Akulov \& R.K. Schumacher, CWU AS $6293=$ HPC 2127 = RKS 116 (holotype CBS H-23832, ex-type culture CPC 34071 = CBS 145039).

Associated with canker on twigs of Acer negundo (Sapindaceae). Sexual morph: Undetermined. Asexual morph: Colonies up to $300 \mu \mathrm{m}$ diam., stromatic, effuse, sporodochial, with partly immersed, basal pseudoparenchymatous stroma, erumpent, black, velvety. Conidiophores $(16-) 21-40(-51) \times 5-12 \mu \mathrm{m}(\bar{x}=31.1 \times 7.6 \mu \mathrm{m}, n=70)$, semi-macronematous, septate, branched, subhyaline, smooth, arising from basal stroma. Conidiogenous cells 3-28 $\mu \mathrm{m}$ long, enteroblastic, annellidic, with 1-2 annellations, integrated, terminal. Conidia (29-)31-36(-42) $\times 11-16.5 \mu \mathrm{m}) \bar{x}=$ $33.5 \times 13.9 \mu \mathrm{m}, n=70$ ), acrogenous, fusiform to obclavate or obpyriform, septate, pale to brown, truncate at base, (2-)3-5(-6) $\mu \mathrm{m}$ diam., with a protruding hilum, rounded at apex, 2-transversely septate, constricted at the septa, minutely echinulate.

Culture characteristics - Colonies on MEA, reaching $2.5 \mathrm{~cm}$ diam. after 2 weeks at $25-30^{\circ} \mathrm{C}$, producing dense mycelium, circular, velvety to woolly, rough margin, white to creamy-grey, with aerial mycelium.

Material examined - RUSSIA, Rostov region, Krasnosulinsky District, Donskoye forestry, artificial forest, on dead and dying attached twigs of Acer negundo (Sapindaceae), 6 April 2016, T.S. Bulgakov, T-1465 (MFLU 16-1759), living culture MFLUCC 16-1183; ibid., Shakhty City, ravine grove near Atukhta river, 5 March 2016, T-1288 (MFLU 16-1582), living culture MFLUCC 16-1157.

Host and distribution - Acer negundo (Russia, Ukraine).

Notes - The BLASTn search of ITS and LSU sequences showed that our strains (MFLUCC 16-1157 and MFLUCC 16-1183) are 99\% similar (ITS = 478/478 bp, LSU = 804/805 bp) to Neodothidotthia negundinicola (CBS 145039). Crous et al. (2019) introduced N. negundinicola, collected from Acer negundo in Ukraine. The morphology of our fresh collections resembles $N$. negundinicola (ex-type: CBS 145039) (Crous et al. 2019) based on its conidial shape and dimension (Table 4). The phylogenetic analyses based on the combined LSU, SSU, ITS and TEF1- 
$\alpha$ sequence dataset show that the strains MFLUCC16-1183 and MFLUCC 16-1157 group with the ex-type strain of $N$. negundinicola (CBS 145039) and has a close relationship with $D$. negundinis and D. robiniae in Dothidotthia (Figs 2, 3; clade E). We, hence, designate Neodothidotthia as a synonym of Dothidotthia and introduce a new combination for the species, Dothidotthia negundinicola. Our new collections are the first records from Russia.


Figure 4 - Dothidotthia negundinicola (MFLU 16-1759). a-c Sporodochia on host surface. $\mathrm{d}$ Vertical section of sporodochium. e-g Conidia attached to the conidiogenous cells. h, i Conidia. j Germinated conidia. Scale bars: $b=1000 \mu \mathrm{m}, \mathrm{c}=500 \mu \mathrm{m}, \mathrm{d}=200 \mu \mathrm{m}, \mathrm{e}-\mathrm{i}=20 \mu \mathrm{m}, \mathrm{j}=40 \mu \mathrm{m}$.

Dothidothia negundinis (Crous) Senwanna, Phookamsak \& K.D. Hyde, comb. nov.

Index Fungorum number: IF556646

Basionym: Thyrostroma negundinis (Berk. \& M.A. Curtis) A.W. Ramaley, Mycotaxon 94: 131 (2006) [2005]

Synonym: Neodothidotthia negundinis (Berk. \& M.A. Curtis) Crous, in Crous et al., Fungal Systematics and Evolution 3: 94 (2019)

Description - See Phillips et al. (2008).

Host and distribution - Acer negundo, Euonymus alatus, Fendlera rupicola (USA). 
Table 4 Morphological comparison of the asexual morph of Thyrostroma, Dothidotthia and related species discussed in this study.

\begin{tabular}{|c|c|c|c|c|c|c|}
\hline \multirow[b]{2}{*}{ Taxon } & \multicolumn{2}{|c|}{ Conidia } & \multirow[b]{2}{*}{$\begin{array}{l}\text { Conidiophore size } \\
(\mu \mathrm{m})\end{array}$} & \multirow{2}{*}{$\begin{array}{l}\text { Conidiogenous } \\
\text { cells (length, } \\
\mu \mathrm{m})\end{array}$} & \multirow[b]{2}{*}{ Host/substrate/Locality } & \multirow[b]{2}{*}{ Reference } \\
\hline & $\begin{array}{l}\text { Size }(\mu \mathrm{m}) \text {, conidial base }(\mu \mathrm{m} \\
\text { diam.) }\end{array}$ & Type \& Septation* & & & & \\
\hline $\begin{array}{l}\text { Dothidotthia } \\
\text { negundinis }\end{array}$ & $18-47 \times 13.5-21.5$ & $\begin{array}{l}\text { Phragmospores, } \\
(1-) 3 ; \text { mostly 2- } \\
\text { septate/0 }\end{array}$ & - & - & $\begin{array}{l}\text { Euonymus alatus (Celastraceae), USA; } \\
\text { Acer negundo (Sapindaceae), USA; } \\
\text { Fendlera rupicola (Hydrangeaceae), } \\
\text { USA }\end{array}$ & $\begin{array}{l}\text { Barr 1989, } \\
\text { Ramaley } 2005\end{array}$ \\
\hline D. negundinicola & $\begin{array}{l}(29-) 31-36(-42) \times 11-16.5, \\
(2-) 3-5(-6)\end{array}$ & Phragmospores, 2/0 & $\begin{array}{l}(16-) 21-40(-51) \times \\
5-12\end{array}$ & $3-28$ & Acer negundo (Sapindaceae), Russia & This study \\
\hline D. negundinicola & $\begin{array}{l}(25-) 30-35(-37) \times(12-) 13- \\
15(-16), 4-5\end{array}$ & Phragmospores, 2/0 & $60-150 \times 7-12$ & $8-15 \times 5-7$ & Acer negundo (Sapindaceae), Ukraine & $\begin{array}{l}\text { Crous et al. } \\
2019\end{array}$ \\
\hline D. robiniae & $\begin{array}{l}(26-) 30-40(-46) \times(10-) 13- \\
16(-18),(2-) 3-5\end{array}$ & $\begin{array}{l}\text { Phragmospores, } 2-3 \\
\text { (mostly 2-septate) } / 0\end{array}$ & $\begin{array}{l}18-) 22-49(-54) \times \\
6-9(-11)\end{array}$ & $7-28$ & $\begin{array}{l}\text { Robinia pseudoacacia and and } R . \\
\text { neomexicana (Fabaceae), Russia }\end{array}$ & This study \\
\hline $\begin{array}{l}\text { Thyrostroma } \\
\text { celtidis }\end{array}$ & $\begin{array}{l}(24-) 27-48(-59) \times 12-19(- \\
21), 4-7\end{array}$ & $\begin{array}{l}\text { Dictyospores, 3-7/2- } \\
5\end{array}$ & $\begin{array}{l}(21-) 25-51(-57) \times \\
3-8\end{array}$ & $9-18$ & $\begin{array}{l}\text { Celtis occidentalis (Cannabaceae), } \\
\text { Russia }\end{array}$ & This study \\
\hline T. cornicola & $\begin{array}{l}(25-) 30-36(-40) \times(12-) 14- \\
17(-26), 5-6\end{array}$ & $\begin{array}{l}\text { Dictyospores, } 1-3 / 0- \\
3\end{array}$ & $10-50 \times 7-10$ & $7-20$ & Cornus officinalis (Cornaceae), Korea & $\begin{array}{l}\text { Crous et al. } \\
2016\end{array}$ \\
\hline T. ephedricola & $25-34 \times 14-22$ & $\begin{array}{l}\text { Dictyospores, } 1-3 / 0- \\
4\end{array}$ & $25-30 \times 4-5$ & - & Ephedra equisetina (Ephedraceae) & $\begin{array}{l}\text { Pem et al. } \\
2019\end{array}$ \\
\hline T. franseriae & $\begin{array}{l}(25-) 28-33(-35) \times(18-) 20- \\
25,8-9\end{array}$ & $\begin{array}{l}\text { Dictyospores; } 1-3 / 2- \\
4\end{array}$ & $10-18 \times 6-11$ & $5-10$ & Franseria sp. (Asteraceae), USA & $\begin{array}{l}\text { Marin-Felix et } \\
\text { al. } 2017\end{array}$ \\
\hline T. jaczewskii & $28-42 \times 13-17$ & $\begin{array}{l}\text { Dictyospores; } 2-6 / 0- \\
1\end{array}$ & $13-16 \times 4-9$ & - & Elaeagnus angustifolia (Elaeagnaceae) & $\begin{array}{l}\text { Pem et al. } \\
2019\end{array}$ \\
\hline T. lycii & $(36-) 39-49(-55) \times 10-17,5-7$ & $\begin{array}{l}\text { Phragmospores, } 1-3 \\
\text { (mostly 3-septate)/0 }\end{array}$ & $\begin{array}{l}(20-) 34-55(-74) \times \\
6-9\end{array}$ & $8-26$ & Lycium barbarum (Solanaceae), Russia & This study \\
\hline T. moricola & $\begin{array}{l}(30-) 33-60(-73) \times(10-) 12- \\
21(-25), 6-9\end{array}$ & $\begin{array}{l}\text { Dictyospores, 3-8/3- } \\
6\end{array}$ & $(28-) 33-50 \times 5-8$ & $9-20$ & Morus alba (Moraceae), Russia & This study \\
\hline T. robiniae & $\begin{array}{l}(33-) 38-50(-53) \times(11-) 13- \\
20,(4-) 5-8\end{array}$ & $\begin{array}{l}\text { Dictyospores, 3-4/0- } \\
3\end{array}$ & $\begin{array}{l}(18-) 23-35(-43) \times \\
4-9\end{array}$ & $8-16$ & $\begin{array}{l}\text { Robinia pseudoacacia (Fabaceae), } \\
\text { Russia }\end{array}$ & This study \\
\hline T. styphnolobii & $\begin{array}{l}(26-) 30-38 \times(11-) 13-18(- \\
22),(4-) 5-7\end{array}$ & $\begin{array}{l}\text { Dictyospores, } 2-3 \\
\text { (mostly 3-septate)/0- } \\
2\end{array}$ & $\begin{array}{l}(14-) 18-33(-35) \times \\
4-9\end{array}$ & $5-25$ & $\begin{array}{l}\text { Styphnolobium japonicum (Ulmaceae), } \\
\text { Russia }\end{array}$ & This study \\
\hline T. tiliae & $\begin{array}{l}(41-) 50-77(-88) \times(12-) 15- \\
21(-23),(3-) 5-6(-9)\end{array}$ & $\begin{array}{l}\text { Dictyospores, 3-7/0- } \\
5\end{array}$ & $\begin{array}{l}(24-) 27-54(-69) \times \\
(3-) 5-9\end{array}$ & $9-29$ & $\begin{array}{l}\text { Tilia cordata (Malvaceae), Russia; } \\
\text { Ulmus pumila (Ulmaceee), Russia }\end{array}$ & This study \\
\hline T. ulmicola & $\begin{array}{l}(30-) 35-50(-59) \times(12-) 15- \\
20(-26),(3-) 4-5(-7)\end{array}$ & $\begin{array}{l}\text { Dictyospores, 3-7/0- } \\
5\end{array}$ & $\begin{array}{l}(12-) 23-56(-73) \times \\
(3-) 4-8(-10)\end{array}$ & $(6-) 10-13$ & Ulmus pumila (Ulmaceae), Russia & This study \\
\hline T. ulmigenum & $\begin{array}{l}(39-) 42-67(-90) \times(11-) 13- \\
17(-20),(3-) 4-5(-6)\end{array}$ & $\begin{array}{l}\text { Dictyospores, 3-7/0- } \\
3\end{array}$ & $\begin{array}{l}(15-) 25-67(-77) \times \\
3-9\end{array}$ & $3-29$ & Ulmus pumila (Ulmaceae), Russia & This study \\
\hline
\end{tabular}


Notes - Crous et al. (2019) proposed a new combination under Neodothidotthia negundinis based on collections of Ramaley (2005) which were identified as Thyrostroma negundinis (Berk. \& M.A. Curtis) A.W. Ramaley (collection no. A.W. Ramaley 0403, 0411 and 0414). Ramaley (2005) introduced a new combination as Thyrostroma negundinis and treated Coryneum negundinis Berk. \& M.A. Curtis as a basionym of T. negundinis without studying the type. She also proposed Dothidotthia aspera (Ellis \& Everh.) M.E. Barr as the sexual morph of Thyrostroma negundinis based on cultural characteristics. However, the connection between Thyrostroma negundinis and Dothidotthia aspera has not yet been proven. Phillips et al. (2008) followed Ramaley (2005) and treated Ramaley's collections (A.W. Ramaley 0403, 0411 and 0414) as Dothidotthia aspera based on phylogenetic analysis of a combined SSU and LSU sequence dataset. However, Crous et al. (2019) compared the morphology of these specimens with the holotype of Amphisphaeria aspera Ellis \& Everh. (basionym of Dothidotthia aspera) and mentioned that these collections are not conspecific with Dothidotthia aspera. Based on phylogenetic analysis coupled with morphological distinctiveness, Crous et al. (2019) treated these collections as Neodothidotthia negundinis in the new genus Neodothidotthia. In this study, N. negundinis isolates (CPC 12928, CPC 12930, CPC 12932 and CPC 12933), obtained from Ramaley's collection (no. 0403, 0411 and 0414), form a sister subclade with Dothidotthia negundinicola and cluster with other known Dothidotthia species in Dothidotthiaceae. We, therefore, synonymized Neodothidotthia negundinis under Dothidotthia as D. negundinis based on Ramaley's collections. However, we do not treat Coryneum negundinis as the basionym of this species and do not reinstate these collections as Dothidotthia aspera due to lack of their type studies. Further studies of type specimens, as well as their epitypes are needed to clarify their taxonomic boundaries.

Dothidotthia robiniae Senwanna, Wanas., Bulgakov, Phookamsak \& K.D. Hyde, sp. nov. Fig. 5 Index Fungorum number: IF556526; Facesoffungi number: FoF06140

Etymology - Named after the host genus Robinia, from which this species was isolated.

Holotype - MFLU 16-1663

Associated with canker on branches and twigs of Robinia neomexicana and $R$. pseudoacacia (Fabaceae). Sexual morph: Undetermined. Asexual morph: Colonies 110-710 $\mu \mathrm{m}$ diam., partly immersed, stromatic, sporodochial, effuse, with partly immersed to erumpent, basal pseudoparenchymatous stroma, black, velvety. Conidiophores $(18-) 22-49(-54) \times 6-9(-11) \mu \mathrm{m}$ ) $\bar{x}=34.5 \times 8 \mu \mathrm{m}, n=60$ ), semi-macronematous, short, compactly packed, septate, subhyaline, smooth, arising from the basal stroma. Conidiogenous cells 7-28 $\mu \mathrm{m}$ long, enteroblastic, annellidic, integrated, terminal. Conidia (26-)30-40(-46) $\times(10-) 13-16(-18) \mu \mathrm{m}) \bar{x}=34.3 \times$ $14.6 \mu \mathrm{m}, n=120$ ), acrogenous, fusiform, obclavate or obpyriform, pale to golden brown, truncate at base, (2-)3-5 $\mu \mathrm{m}$ diam., rounded at apex, 2-3-septate (mostly 2-septate), constricted at the septa, rough-walled, minutely echinulate.

Culture characteristics - Colonies on MEA, reaching $2.5 \mathrm{~cm}$ diam. after 2 weeks at 25$30^{\circ} \mathrm{C}$, producing dense mycelium, circular, velvety, rough margin, greenish-brown, lacking aerial mycelium. Colonies on PDA, slow-growing, reaching $1 \mathrm{~cm}$ diam. after 2 weeks at 25$30^{\circ} \mathrm{C}$, producing dense mycelium, rough margin, white, lacking aerial mycelium.

Material examined - RUSSIA, Rostov region, Shakhty City, near the spoil tip of former coal mine "Proletarian Dictature", on dead twigs of Robinia pseudoacacia (Fabaceae), 24 March 2016, T.S. Bulgakov, T-1369 (MFLU 16-1663, holotype), ex-type living culture MFLUCC 161175; Rostov region, Krasnosulinsky District, Donskoye forestry, artificial forest, on dead attached twigs of Robinia neomexicana (Fabaceae), 6 April 2016, T.S. Bulgakov, T-1410 (MFLU 16-1704), living culture MFLUCC 16-1177; Rostov region, Shakhty City, artificial forest near Grushevka river, on dead twigs of Robinia pseudoacacia (Fabaceae), 14 May 2016, T.S. Bulgakov, T-1504 (MFLU 16-1798), living culture MFLUCC 16-1185; Rostov region, Shakhty City, Solyonaya balka (Salty gully), artificial forest, on dead twigs of Robinia 
pseudoacacia (Fabaceae), 14 May 2016, T.S. Bulgakov, T-1509 (MFLU 16-1803), living culture MFLUCC 18-0692.

Host and distribution - Robinia neomexicana and R. pseudoacacia (Russia).

Notes - Based on the NCBI BLASTn search of ITS sequence data, the closest match of Dothidotthia robiniae is D. aspera (CPC 12933; 99\% similarity). Multi-gene phylogenetic analyses (Figs 2, 3) show that four isolates of D. robiniae (MFLUCC 16-1175, MFLUCC 161177, MFLUCC 16-1185 and MFLUCC 18-0692) form a distinct lineage (clade E), sister to $D$. negundinis and $D$. negundinicola. Dothidotthia robiniae is morphologically similar to $D$. negundinis (CPC 12930 and CPC12933) and D. negundinicola (MFLUCC 16-1157 and MFLUCC 16-1183) in conidial shape. However, these species differ in their conidial dimensions (Table 4). A comparison of ITS nucleotides shows that $D$. robiniae is not significantly different from $D$. negundinis (7/478 bp (1.46\%)) and D. negundinicola (5/478 bp (1.05\%)). However, a comparison of RPB2 sequence data shows that $D$. robiniae differs from $D$. negundinicola in 31/1057 bp (2.93\%). Thus, we introduce $D$. robiniae as a new species in Dothidotthia.
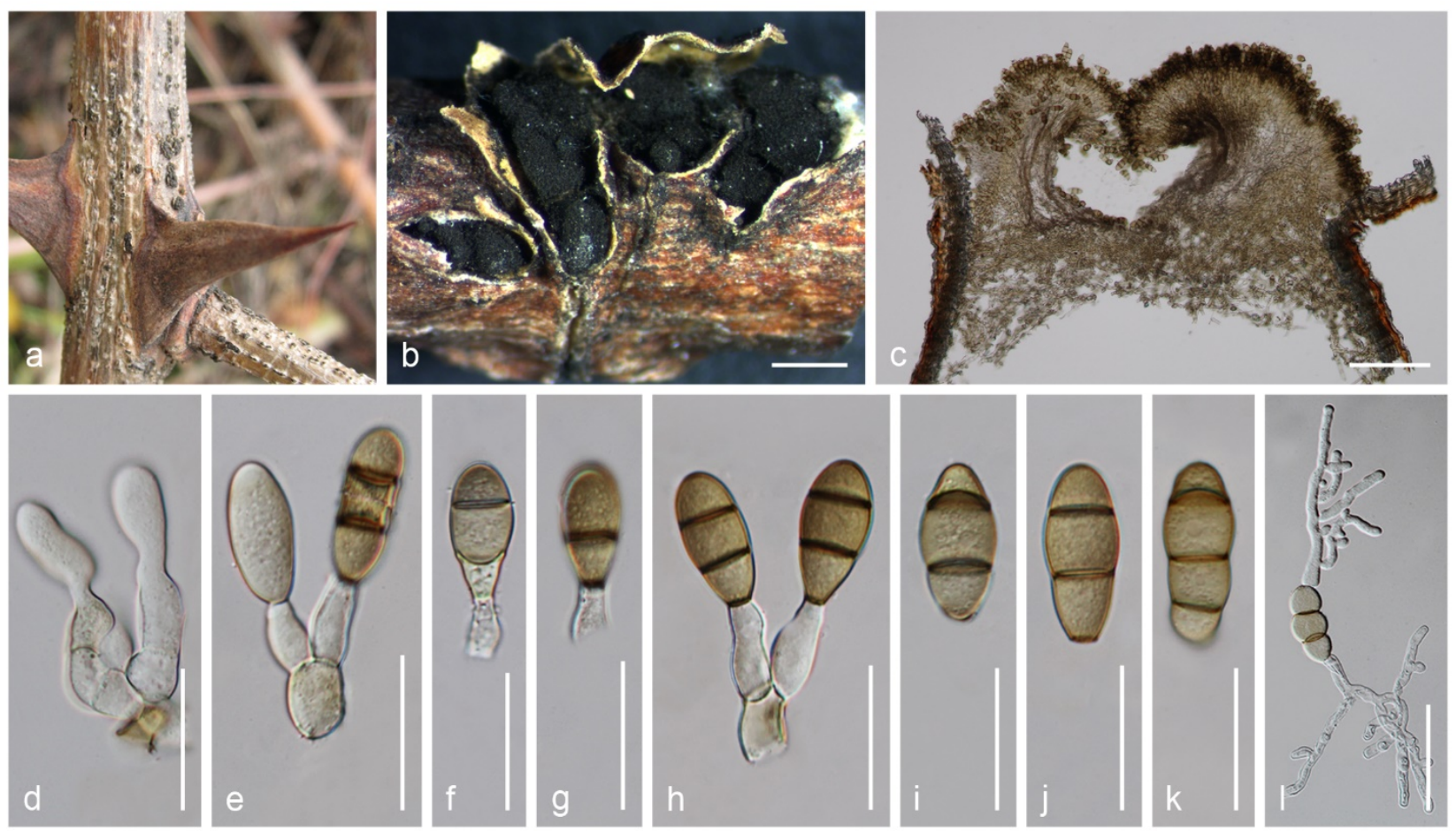

Figure 5 - Dothidotthia robiniae (MFLU 16-1663, holotype). a, b Sporodochia on host surface. c Vertical section of sporodochium. d Conidiogenesis. e-h Conidia attach to conidiogenous cells. i-k Conidia. l Germinated conidia. Scale bars: $b=1000 \mu \mathrm{m}, \mathrm{c}=200 \mu \mathrm{m}, \mathrm{d}-\mathrm{l}=30 \mu \mathrm{m}$.

Thyrostroma Höhn., Sitzungsberichte der Kaiserlichen Akademie der Wissenschaften Math.naturw. Klasse Abt. I 120: 472 (1911)

Saprobic, pathogenic on leaves and wood in terrestrial habitats (Fig. 1). Sexual morph: Ascostromata pseudothecial, immersed, raised, erumpent to superficial, breaking through host surface, ruptured, rounded, elongate, lenticular, or irregular in shape, uni- to multi-loculate, glabrous, ostiolate, apapillate. Ascomata immersed in ascostroma, dark brown to black, clustered, gregarious, rarely solitary, globose to subglobose, ostiole central, apapillate. Peridium thin- to thick-walled of unequal thickness, thicker at the apex, thinner at the base, with several cell layers of textura angularis, outer layer comprising brown to black, inner layer comprising hyaline to pale brown cells. Hamathecium composed of dense, hyaline, septate pseudoparaphyses. Asci 8-spored, bitunicate, fissitunicate, clavate, short pedicellate, apically 
rounded, with well-developed ocular chamber. Ascospores, pale brown, fusiform to ellipsoidal, with rounded ends, 1-septate, constricted at the septum. Asexual morph: Colonies stromatic, sporodochial, immersed to erumpent, breaking through host surface, ruptured, convex to applanate, or pulvinate, lenticular, or irregularly dehiscent, dark brown to black. Conidiophores macronematous, cylindrical to subcylindrical, septate, branched, hyaline to brown, arising from basal pseudoparenchymatous sporodochia. Conidiogenous cells holoblastic, monoblastic, polyblastic, integrated, terminal. Conidia acrogenous, straight or curved, variable in shape, clavate, ellipsoidal, obpyriform, subglobose, or oblong to subcylindric-clavate, phragmosporous to muriform, rounded at the apex, tapered or truncate at the base, with 1-8 transverse septa, and 0-6 longitudinal septa, pale to dark brown, constricted at the septa, rough-walled, echinulate.

Type species - Thyrostroma compactum (Sacc.) Höhn.

Notes - Thyrostroma was previously considered as the asexual morph of Dothidotthia (Ramaley 2005, Phillips et al. 2008). Phylogenetic analysis of LSU sequence data indicated that Thyrostroma and Dothidotthia are not congeneric (Crous et al. 2016, Marin-Felix et al. 2017). Currently, there are 15 epithets listed in this genus (Index Fungorum 2019). However, there are 165 sequences available in GenBank, comprising T. compactum, T. cornicola, T. franseriae and our sequences in this study. In our phylogenetic analyses, Thyrostroma species form a strongly supported clade (77\% ML, 97\% MP, 1.00 PP; Fig. 2) distinct from Dothidotthia in Dothidotthiaceae. Further, we introduce eight novel species, viz. T. celtidis, T. lycii, T. moricola, T. robiniae, $T$. styphnolobii, $T$. tiliae, $T$. ulmigenum and $T$. ulmicola. We also report the sexual morph of T. ulmicola and it is the first record of sexual morph in Thyrostroma.

Thyrostroma celtidis Senwanna, Wanas., Bulgakov, Phookamsak \& K.D. Hyde, sp. nov. Fig. 6 Index Fungorum number: IF556527; Facesoffungi number: FoF06141

Etymology - Named after the host genus on which it occurs, Celtis.

Holotype - MFLU 16-1800

Associated with canker on twigs of Celtis occidentalis (Cannabaceae). Sexual morph: Undetermined. Asexual morph: Colonies stromatic, sporodochial, comprising pseudoparenchymatous cells at basal stroma, erumpent through host surface, ruptured, applanate, circular to elliptical, or lenticular dehiscent, black, velvety. Conidiophores (21-)25-51(-57) $\times 3-$ $8 \mu \mathrm{m}) \bar{x}=35.7 \times 5.3 \mu \mathrm{m}, n=25$ ), macronematous, septate, branched, hyaline, smooth, arising from the basal stroma. Conidiogenous cells 9-18 $\mu \mathrm{m}$ long, holoblastic, integrated, terminal. Conidia (24-)27-48(-59) $\times 12-19(-21) \mu \mathrm{m}) \bar{x}=35.8 \times 15.9 \mu \mathrm{m}, n=75)$, acrogenous, straight or curved, variable in shape, usually clavate to obpyriform, tapered to the base, truncate at base, 4-7 $\mu \mathrm{m}$ diam., rounded at apex, muriform, (3-)5-6 transverse septa, with 2-5 longitudinal septa, constricted at the septa, pale to dark brown, rough-walled, minutely echinulate.

Culture characteristics - Colonies on PDA, slow growing, reaching $1 \mathrm{~cm}$ diam. after 3 weeks at $25-30^{\circ} \mathrm{C}$, producing dense mycelium, raised to pulvinate, rough margin, dark brown, lacking aerial mycelium.

Material examined - RUSSIA, Rostov region, Shakhty City, Solyonaya balka (Salty gully), artificial forest, on dead and dying attached twigs of Celtis occidentalis, 21 May 2015, T.S. Bulgakov, T-1506 (MFLU 16-1800, holotype), ex-type living culture MFLUCC 16-1186.

Host and distribution - Celtis occidentalis (Russia).

Notes - In the NCBI BLASTn search of ITS sequences, Thyrostroma celtidis most closely matches T. cornicola Crous \& H.D. Shin and T. compactum with 99\% similarity. Phylogenetic analyses of multi-gene sequence dataset show that $T$. celtidis forms a separate lineage with $T$. cornicola and T. compactum and other Thyrostroma species (Figs 2, 3). A comparison of ITS nucleotides shows that $T$. celtidis differs from $T$. compactum in 19/481 bp (3.95\%), and also different from $T$. cornicola in 6/479 bp (1.25\%). They are different in conidial dimension and conidial morphology (Table 4). Thyrostroma celtidis has a close phylogenetic relationship and similar morphological characters with T. jaczewskii (B. Suttton) D. Pem, Bulgakov, Jeewon \& 
K.D. Hyde (Pem et al. 2019). However, these two species occurred on different hosts and countries and their conidial dimension and septation were different. A comparison of ITS nucleotides shows that $T$. celtidis differs from $T$. jaczewskii in $17 / 479$ bp (3.52\%). The conidial morphology of $T$. celtidis is similar to $T$. ulmicola but $T$. celtidis differs from $T$. ulmicola in having shorter conidia and a different number of transverse and longitudinal septa (Table 4).


Figure 6 - Thyrostroma celtidis (MFLU 16-1800, holotype). a, b Sporodochia on host surface. c Vertical section of sporodochium. $\mathrm{d}-\mathrm{f}$ Conidia attached to conidiogenous cells. g-k Conidia with variable shape. Scale bars: $b=500 \mu \mathrm{m}, \mathrm{c}=300 \mu \mathrm{m}, \mathrm{d}-\mathrm{k}=30 \mu \mathrm{m}$.

Thyrostroma lycii Senwanna, Wanas., Bulgakov, Phookamsak \& K.D. Hyde, sp. nov.

Fig. 7 Index Fungorum number: IF556528; Facesoffungi number: FoF06142

Etymology - Named after the host genus on which it occurs, Lycium.

Holotype - MFLU 16-1642

Associated with canker on twigs of Lycium barbarum (Solanaceae). Sexual morph: Undetermined. Asexual morph: Colonies 260-370 $\mu \mathrm{m}$ diam. sporodochia, stromatic, sporodochial, comprising pseudoparenchymatous cells at basal stroma, partly immersed, becoming erumpent through host epidermis, ruptured, applanate, black, velvety. Conidiophores $(20-) 34-55(-74) \times 6-9 \mu \mathrm{m}) \bar{x}=45.2 \times 7.8 \mu \mathrm{m}, n=10)$, compactly arranged, macronematous, septate, branched, hyaline, smooth. Conidiogenous cells 8-26 $\mu \mathrm{m}$ long, holoblastic, monoblastic, integrated, terminal. Conidia (36-)39-49(-55) $\times 10-17 \mu \mathrm{m}) \bar{x}=43 \times 13.7 \mu \mathrm{m}, n=50$ ), acrogenous, slightly curved, ellipsoidal to clavate, pale to golden brown, truncate at base, 5-7 $\mu \mathrm{m}$ diam., rounded at apex, 1-4-septate (mostly 3-septate), constricted at the septa, minutely echinulate.

Culture characteristics - Colonies on PDA, reaching $2.5 \mathrm{~cm}$ diam. after 2 weeks at 25$30^{\circ} \mathrm{C}$, producing dense mycelium, rough margin, white at the margin, greenish brown at the centre, lacking aerial mycelium.

Material examined - RUSSIA, Rostov region, Shakhty City, near spoil tip of a former coal mine "Proletarian Dictature", on dead and dying twigs of Lycium barbarum (Solanaceae), 24 March 2016, T.S. Bulgakov, T-1348 (MFLU 16-1642, holotype), ex-type living culture MFLUCC 16-1170. 
Host and distribution - Lycium barbarum (Russia).

Notes - In the NCBI BLASTn search of ITS sequence, Thyrostroma lycii has the closest match with $T$. compactum (CBS 335.37) with 99\% similarity and is also similar to T. compactum (D5/5c; fungal endophyte) and T. cornicola (CPC 25427) with 97\% similarity. Thyrostroma lycii is introduced as a new species based on morphological characters and phylogenetic evidence. Phylogenetic analyses of the combined LSU, SSU, ITS and TEF1- $\alpha$ sequence dataset show that T. lycii forms a distinct lineage closely related to T. compactum, T. ulmigenum, T. tiliae and T. ulmicola (Figs 2, 3; clade A). Thyrostroma lycii is different from the other Thyrostroma in having phragmosporous conidia, while others have muriform conidia.

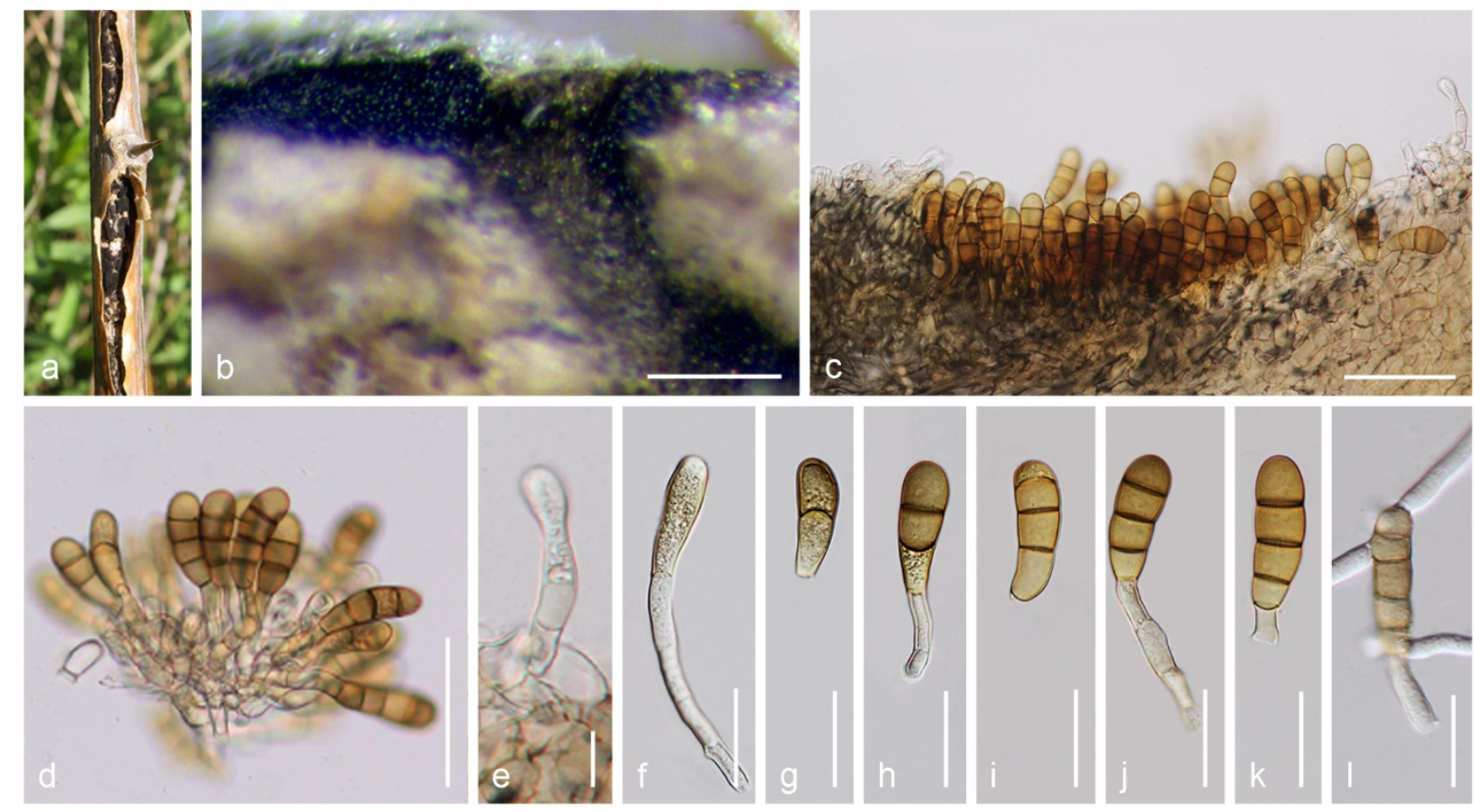

Figure 7 - Thyrostroma lycii (MFLU 16-1642, holotype). a, b Sporodochia on host surface. c Vertical section of sporodochium. d Conidia attached with conidiogenous cells. e Conidiogenous cell. $\mathrm{f}-\mathrm{k}$ Different stages of conidial development and conidia. $\mathrm{l}$ Germinated conidium. Scale bars: $\mathrm{a}=1000 \mu \mathrm{m}, \mathrm{b}, \mathrm{c}=200 \mu \mathrm{m}, \mathrm{d}-\mathrm{m}=30 \mu \mathrm{m}$.

Thyrostroma moricola Senwanna, Wanas., Bulgakov, Phookamsak \& K.D. Hyde, sp. nov. Fig. 8 Index Fungorum number: IF556529; Facesoffungi number: FoF06143

Etymology - Named after the host genus Morus, from which this species was isolated.

Holotype - MFLU 16-1795

Associated with canker on twigs of Morus alba (Moraceae). Sexual morph: Undetermined. Asexual morph: Colonies 285-1295 $\mu \mathrm{m}$ diam., stromatic, sporodochial, with pseudoparenchymatous cells at basal stroma, partly immersed, becoming erumpent through host epidermis, ruptured, lenticular or irregular in shape, applanate, black, velvety. Conidiophores (28-)33-50 × 5-8 $\mu \mathrm{m}) \bar{x}=35.6 \times 6.4 \mu \mathrm{m}, n=6$ ), compactly arranged, macronematous, septate, pale yellowish to pale brown, smooth. Conidiogenous cells 9-20 $\mu \mathrm{m}$ long, holoblastic, polyblastic, sympodial (Figs 8e, f), integrated, terminal. Conidia (30-)33-60(-73) $\times(10-) 12-$ $21(-25) \mu \mathrm{m}) \bar{x}=45 \times 17.2 \mu \mathrm{m}, n=80$ ), acrogenous, muriform, with several sectors, straight or slightly curved, varied in shape but commonly broadly subglobose to clavate, brown to dark brown, 3-8 transverse septa, with 3-6 longitudinal septa, rounded at the apex, truncate at the base, 6-9 $\mu \mathrm{m}$ diam., smooth. 
Material examined - RUSSIA, Rostov region, Shakhty City, Solyonaya balka (salty gully), artificial forest, on dead twigs of Morus alba (Moraceae), 21 May 2015, T.S. Bulgakov, T-1501 (MFLU 16-1795, holotype).

Host and distribution - Morus alba (Russia).


Figure 8 - Thyrostroma moricola (MFLU 16-1795, holotype). a-c Sporodochia on host surface. d Vertical section of sporodochium. e, f Polyblastic conidiogenesis. g, k, m Conidia with conidiogenous cells. h, i, j, n Conidia. Scale bars: b, $c=1000 \mu \mathrm{m}, \mathrm{d}=200 \mu \mathrm{m}, \mathrm{e}-\mathrm{n}=20 \mu \mathrm{m}$.

Notes - Thyrostroma moricola is associated with canker on twigs of Morus alba in Russia. The species can be distinguished from other Thyrostroma species based on phylogenetic analyses and characteristics of conidial development, size and septation. The species is similar to $T$. celtilis in conidial shape but differs in size of conidiophores and conidia and numbers of conidial septation (Table 4). Unlike in the others, the conidial development of this species is polyblastic and sympodial (Figs 8e, f). In phylogenetic analyses, T. moricola has a close relationship with $T$. styphnolobii (Figs 2, 3; clade A). However, these taxa are different in conidial shape and size (Table 4). In the NCBI BLASTn search of LSU and ITS sequences, T. moricola most closely 
matches T. cornicola (99\% similarity). A comparison of ITS and TEF1- $\alpha$ nucleotide base pairs shows that $T$. moricola is not significantly different from $T$. celtidis and $T$. styphnolobii. However, the species is significantly different from $T$. celtidis and $T$. styphnolobii based on comparison of TUB2 gene (9/346 bp (2.60\%) and 15/346 bp (4.35\%), respectively). We, therefore, introduce $T$. moricola as a new species.

Thyrostroma robiniae Senwanna, Wanas., Bulgakov, Phookamsak \& K.D. Hyde, sp. nov. Fig. 9 Index Fungorum number: IF556530; Facesoffungi number: FoF06144

Etymology - Named after the host genus Robinia, from which this species was isolated.

Holotype - MFLU 18-0631

Associated with canker on twigs of Robinia pseudoacacia (Fabaceae). Sexual morph: Undetermined. Asexual morph: Colonies stromatic, with pseudoparenchymatous cells at basal stroma, partly immersed, erumpent through host epidermis, black, velvety, lenticular or triangular dehiscent. Conidiophores (18-)23-35(-43) $\times 4-9 \mu \mathrm{m}) \bar{x}=28.7 \times 6.5 \mu \mathrm{m}, n=10$ ), compactly arranged, macronematous, septate, pale brown, smooth, reduced to conidiogenous cells. Conidiogenous cells 8-16 $\mu \mathrm{m}$ long, holoblastic, monoblastic, integrated, terminal. Conidia (33-)38-50(-53) $\times(11-) 13-20 \mu \mathrm{m}) \bar{x}=44 \times 15.7 \mu \mathrm{m}, n=30$ ), acrogenous, slightly curved, oblong to clavate, pale to dark brown, truncate at the base, (4-)5-8 $\mu \mathrm{m}$ diam., rounded at the apex, muriform, 3-4 transverse septa (mostly 3-septate), with 0-3 longitudinal septa, constricted at the septa, rough-walled, minutely echinulate.

Culture characteristics - Colonies on PDA, slow-growing, reaching $1 \mathrm{~cm}$ diam. after 3 weeks at $25-30^{\circ} \mathrm{C}$, producing dense mycelium, raised to pulvinate, rough margin, light brown, lacking aerial mycelium.
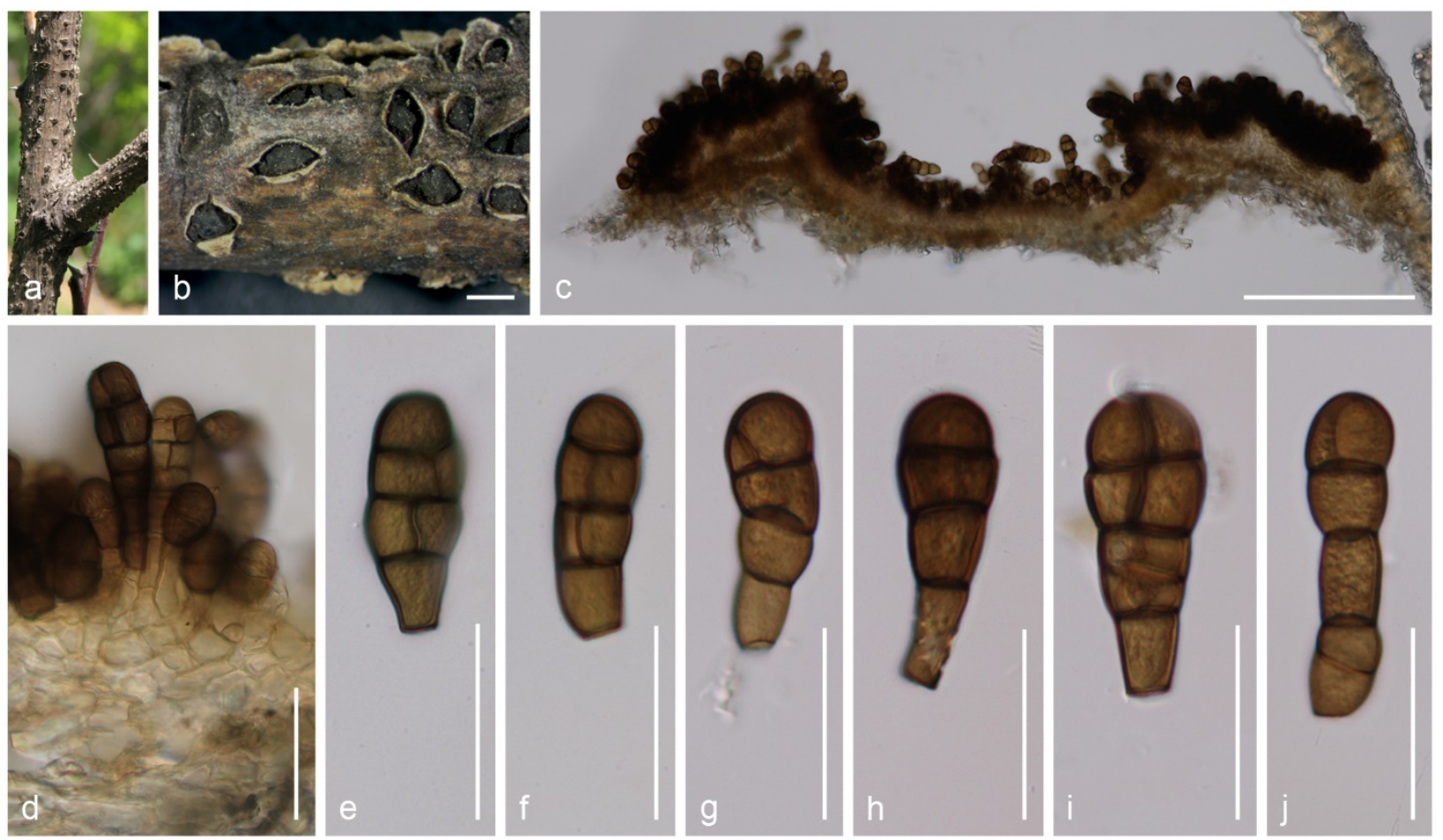

Figure 9 - Thyrostroma robiniae (MFLU 18-0631, holotype). a, b Sporodochia on host surface. c Vertical section of sporodochium. d Conidia attached with conidiogenous cells. e-j Variable in shape of conidia. Scale bars: $b=1000 \mu \mathrm{m}, \mathrm{c}=200 \mu \mathrm{m}, \mathrm{d}-\mathrm{j}=30 \mu \mathrm{m}$.

Material examined - RUSSIA, Rostov region, Shakhty City, artificial forest near Grushevka River, on dead twigs of young trees of Robinia pseudoacacia (Fabaceae), 14 May 
2015, T.S. Bulgakov, T-1504B (MFLU 18-0631, holotype), ex-type living culture MFLUCC 181191.

Host and distribution - Robinia pseudoacacia (Russia).

Notes - In the NCBI BLASTn search of LSU and ITS sequences, Thyrostroma robiniae most closely matches $T$. cornicola with 99\% similarity. Phylogenetic analyses of multi-gene sequence dataset, T. robiniae nested with $T$. celtidis, $T$. moricola and $T$. styphnolobii (Figs 2, 3; clade A). Thyrostroma robiniae is morphologically similar to T. styphnolobii in conidial shape but differs in size of conidia, conidiophores and conidiogenous cells. Whereas, T. robiniae differs from T. celtidis and T. moricola in conidial size and different numbers of septation (Table 4). A comparison of nucleotides of ITS and TEF1- $\alpha$ regions shows that $T$. robiniae is not significantly different from $T$. moricola and T. styphnolobii. However, T. robiniae is distinct from T. moricola and T. styphnolobii in RPB2 and TUB2 regions (180/1057 bp (17.03\%) of RPB2 between $T$. robiniae and $T$. styphnolobii, and 17/347 bp (4.9\%) and 13/346 bp (3.76\%) of TUB2, respectively).

Thyrostroma styphnolobii Senwanna, Wanas., Bulgakov, Phookamsak \& K.D. Hyde, sp. nov.

Index Fungorum number: IF556531; Facesoffungi number: FoF06145

Fig. 10

Etymology - Named after the host genus Styphnolobium, from which this species was isolated.

Holotype - MFLU 16-1619

Associated with canker and necrosis on branches and twigs of Styphnolobium japonicum (Fabaceae). Sexual morph: Undetermined. Asexual morph: Colonies 230-540 $\mu \mathrm{m}$ diam. sporodochia, effuse, stromatic, sporodochial, with pseudoparenchymatous at the base of stroma, arranged in textura angularis to textura prismatica, partly immersed to erumpent, black, velvety, lenticular, trapezoid or triangular dehiscent. Conidiophores $(14-) 18-33(-35) \times 4-9 \mu \mathrm{m}) \bar{x}=28.2$ $\times 6.5 \mu \mathrm{m}, n=20$ ), semi-macronematous, septate, branched, hyaline to pale brown, smooth, compactly arranged. Conidiogenous cells 5-25 $\mu \mathrm{m}$ long, holoblastic, monoblastic, integrated, terminal. Conidia (26-)30-38 $\times(11-) 13-18(-22) \mu \mathrm{m}) \bar{x}=33.5 \times 14.7 \mu \mathrm{m}, n=25)$, acrogenous, subglobose to oblong, subclavate, pale to dark brown, truncate at the base, (4-)5-7 $\mu \mathrm{m}$ diam., rounded at the apex, muriform, with 2-3 transverse septa (mostly 3-septate), 0-2 longitudinal septa, slightly constricted at the septa, rough-walled, minutely echinulate.

Culture characteristics - Colonies on MEA, reaching $3 \mathrm{~cm}$ diam. after 2 weeks at $25-30^{\circ} \mathrm{C}$, producing dense mycelium, surface dull and smooth, velvety, rough margin, light brown to greyish, lacking aerial mycelium. Colonies on PDA, reaching $1.5 \mathrm{~cm}$ diam. after 3 weeks at 25$30^{\circ} \mathrm{C}$, producing dense mycelium, flat, slightly raised, velvety, rough margin, light brown at the centre, dark brown to black at the margin, with aerial mycelium.

Material examined - RUSSIA, Rostov region, Shakhty City, Alexandrosky Park, on dying and dead twigs of Styphnolobium japonicum (Fabaceae), 14 March 2016, T.S. Bulgakov, T-1325 (MFLU 16-1619, holotype), ex-type living culture MFLUCC 16-1160.

Host and distribution - Styphnolobium japonicum (Russia).

Notes - In the NCBI BLASTn search, Thyrostroma styphnolobii closely matches $T$. cornicola (100\% and 99\% similarity in ITS and LSU sequences, respectively) and T. compactum (99\% similarity in both ITS and LSU sequences). Based on the multi-gene phylogenetic analyses, T. styphnolobii forms a stable lineage, sister to T. moricola in analyses 1 and 2 (Figs 2, 3; clade A) and closely related to T. cornicola, T. franseriae and T. ephedricola. A comparison of ITS nucleotides shows that $T$. styphnolobii differs from $T$. cornicola, $T$. franseriae, $T$. moricola and $T$. ephedricola in 2/452 bp (0.44\%), 3/469 bp (0.64\%), 2/479 bp (0.42\%) and 5/479 bp (1.04\%), respectively. Our attempts to obtain RPB2 for T. styphnolobii was unsuccessful. However, T. styphnolobii and T. moricola are different in a comparison of TEF1- $\alpha$ and TUB2 nucleotides (9/780 bp (1.15\%) of TEF1- $\alpha$ and 15/346 bp (4.33\%) of TUB2). Therefore, we 
introduce $T$. styphnolobii as a new species, based on different type of conidiogenesis, its comparatively smaller conidia and different numbers of transverse and longitudinal septa from $T$. cornicola, T. franseriae and T. moricola (Table 4), and its phylogenetic position.


Figure 10 - Thyrostroma styphnolobii (MFLU 16-1619, holotype). a-c Sporodochia on host surface. d Vertical section of sporodochium. e Conidiogenesis. $\mathrm{f}-\mathrm{i}$ Variable in shape of conidia attached with conidiogenous cells. $\mathrm{m}, \mathrm{n}$ Germinated conidium. Scale bars: $\mathrm{b}=1000 \mu \mathrm{m}$, c, $\mathrm{d}=200 \mu \mathrm{m}, \mathrm{e}-\mathrm{l}=30 \mu \mathrm{m}, \mathrm{m}, \mathrm{n}=60 \mu \mathrm{m}$.

Thyrostroma tiliae Senwanna, Wanas., Bulgakov, Phookamsak \& K.D. Hyde, sp. nov.

Fig. 11

Index Fungorum number: IF556532; Facesoffungi number: FoF06146

Etymology - Named after the host genus on which it occurs, Tilia.

Holotype - MFLU 16-1740

Associated with canker on barks, branches and twigs of Tilia cordata (Malvaceae). Sexual morph: Undetermined. Asexual morph: Colonies 205-780 $\mu \mathrm{m}$ diam. sporodochia, stromatic, sporodochial, pseudoparenchymatous at the base of stroma, partly immersed to erumpent through host epidermis, ruptured, pulvinate; with rounded to lenticular, or irregularly dehiscent, black, velvety, gnarled. Conidiophores (24-)27-54(-69) $\times(3-) 5-9 \mu \mathrm{m}) \bar{x}=40 \times 6.7 \mu \mathrm{m}, n=$ 40), semi-macronematous, septate, branched, hyaline to pale brown, smooth, arising from pseudoparenchymatous stroma. Conidiogenous cells 9-29 $\mu \mathrm{m}$ long, holoblastic, annellidic, integrated, terminal. Conidia (41-)50-77(-88) $\times(12-) 15-21(-23) \mu \mathrm{m}) \bar{x}=62.9 \times 18.1 \mu \mathrm{m}, n=$ 120), acrogenous, cylindrical to ellipsoidal, or subclavate to vermiform, pale to brown, truncate at base, (3-)5-6(-9) $\mu \mathrm{m}$ diam., rounded at apex, muriform, 3-7-transversely septate, with 0-5longitudinally septate at the $2^{\text {nd }}$ to the $4^{\text {th }}$ cells from above, constricted at the septa, rough-walled, echinulate.

Culture characteristics - Colonies on MEA, reaching $3 \mathrm{~cm}$ diam. after 2 weeks at $25-30^{\circ} \mathrm{C}$, producing dense mycelium, flat, surface dull and smooth, rough margin, white to greyish becoming dark brown or greenish-brown when mature, reverse dark brown to black at 
centre, white at the margin, with aerial mycelium.


Figure 11 - Thyrostroma tiliae (MFLU 16-1740, holotype). a, b Sporodochia on host surface. c Vertical section of sporodochium. d, e, h Conidia attached with conidiogenous cells. f-g Conidia. i Germinated conidia. Scale bars: $b=500 \mu \mathrm{m}, \mathrm{c}=200 \mu \mathrm{m}, \mathrm{d}-\mathrm{h}=30 \mu \mathrm{m}$, $\mathrm{i}=60 \mu \mathrm{m}$.

Material examined - RUSSIA, Rostov region, Krasnosulinsky District, Donskoye forestry, artificial forest, on dead and dying twigs of Tilia cordata (Malvaceae), 6 April 2016, T.S. Bulgakov, T-1446 (MFLU 16-1740, holotype), ex-type living culture, MFLUCC 16-1178; ibids., on dead and dying twigs of Tilia cordata (Malvaceae), 6 April 2016, T.S. Bulgakov, T-1387 (MFLU 16-1681), living culture, MFLUCC 16-1176; Rostov region, Krasnosulinsky District, Donskoye forestry, artificial forest, on dead twigs and bark of Ulmus pumila (Ulmaceae), 6 April 2016, T.S. Bulgakov, T-1454B (MFLU 18-0628), living culture MFLUCC 16-1180; Rostov region, Shakhty City, Alexandrovsky Park, on dead and dying twigs of Tilia cordata (Malvaceae), 1 May 2015, T.S. Bulgakov, T-1519, (MFLU 16-1813), living culture, MFLUCC 16-1188.

Host and distribution - Tilia cordata, Ulmus pumila (Russia).

Notes - In the BLASTn search of the ITS sequence, Thyrostroma tiliae has a $98 \%$ (469/480) similarity with T. compactum (CBS 335.37; GenBank KY905670) and a 96\% (462/479) similarity with T. compactum (isolate D5/5C; GenBank MG020345) and T. cornicola (CBS 141280; GenBank KX228248). In this study, we refer four strains to T. tiliae viz. MFLUCC 16-1180, MFLUCC 16-1176, MFLUCC 16-1178 and MFLUCC 16-1188. These four strains form a single lineage with high support (97\% ML, 99\% MP, 1.00 PP, Fig. 3; clade A), 
and is sister to T. ulmigenum. Thyrostroma tiliae morphologically resembles T. ulmigenum in its conidial shape. However, their sporodochia and size of the conidia are different (Table 4). A comparison of ITS and TEF1- $\alpha$ nucleotides shows that T. tiliae is different from T. ulmigenum (5/480 bp (1.04\%) of ITS and 10/787 bp (1.27\%) of TEF1- $\alpha$ ). The species is also different from T. ulmigenum in RPB2 and TUB2 regions (55/1062 bp (5.18\%) and 15/342 bp (4.39\%), respectively). In addition, $T$. tiliae differs from $T$. compactum in $11 / 481$ bp (2.28\%) of ITS region. Thyrostroma compactum var. tiliae (Sacc.) Höhn. associated with linden canker has been also reported in Russia and Eastern Europe from the last decades (Kolemasova 1999, Kuz'michev et al. 2001, Sokolova et al. 2006, Mel'nik et al. 2007, Kolganikhina \& Sokolova 2012, Bulgakov et al. 2014, Stravinskienè et al. 2015). However, the species differs from $T$. tiliae in having shorter and narrower conidia (50-55 × 15-16 $\mu \mathrm{m}$, Potebnia 1907). Thus, we introduce Thyrostroma tiliae as a new species.

Thyrostroma ulmicola Senwanna, Wanas., Bulgakov, Phookamsak \& K.D. Hyde, sp. nov.

Index Fungorum number: IF556533; Facesoffungi number: FoF06283

Fig. 12

Etymology - Named after the host genus on which it occurs, Ulmus pumila.

Holotype - MFLU 16-1652

Associated with canker on twigs and bark of Ulmus pumila (Ulmaceae). Sexual morph: Ascostromata pseudothecial, immersed in host epidermis, visible as raised, becoming erumpent to superficial, ruptured, reflexed, stellate, host remnants around the base, ostiolate, apapillate. Ascomata 180-365 $\mu \mathrm{m}$ diam., 135-310 $\mu \mathrm{m}$ high, immersed in ascostroma, dark brown to black, clustered, gregarious, rarely solitary, globose to subglobose, uni- to multi-loculate, ostiole central, apapillate. Peridium thin- to thick-walled, of unequal thickness, thicker at the apex, composed of 9-11 layers of pale brown to dark brown, or black, pseudoparenchymatous cells, arranged in textura angularis, 95-100 $\mu \mathrm{m}$; thinner at the base, 45-60 $\mu \mathrm{m}$, composed of 5-6 layers of brown to black cells, of textura angularis. Hamathecium composed of dense, filamentous, 3-5 $\mu \mathrm{m}$ wide, hyaline, septate pseudoparaphyses. Asci (71-)113-190(-200) × (13)19-25(-27) $\mu \mathrm{m}(\bar{x}=148 \times 21.8 \mu \mathrm{m}, n=25)$, 8-spored, bitunicate, fissitunicate, clavate, short pedicellate, with knob-like to truncate pedicel, apically rounded, with a well-developed ocular chamber. Ascospores (22-)25-33(-36) $\times(7-) 9-16 \mu \mathrm{m}(\bar{x}=29.8 \times 13.2 \mu \mathrm{m}, n=28)$, overlapping 1-2-seriate, pale brown, fusiform to ellipsoidal with rounded ends, 1-septate, constricted at the septum, widest at above cell, rough-walled, finely verruculose. Asexual morph: Colonies 150-900 $\mu \mathrm{m}$ diam., sporodochia, partly immersed, or effuse, stromatic, with pseudoparenchymatous basal stroma, erumpent through host epidermis, pulvinate to applanate, black, velvety, with lenticular or irregularly dehiscent. Conidiophores (12-)23-56(-73) × (3-)4$8(-10) \mu \mathrm{m}) \bar{x}=40.3 \times 6.1 \mu \mathrm{m}, n=300)$, macronematous, erect, compactly packed, septate, branched, hyaline to pale brown, smooth. Conidiogenous cells (6-)10-13 $\mu \mathrm{m}$ long, holoblastic, monoblastic, annellidic, with 1-2 annellations, integrated, terminal. Conidia (30-)35-50(-59) $\times$ $(12-) 15-20(-26) \mu \mathrm{m}) \bar{x}=42 \times 18.1 \mu \mathrm{m}, n=420)$, acrogenous, ellipsoidal to obovoid, subclavate, muriform, pale to dark brown, truncate at base, (3-)4-5(-7) $\mu \mathrm{m}$ diam., rounded at apex, 3-7-transverse septate, with longitudinally 0-5-septate, constricted at the septa, roughwalled, minutely echinulate.

Culture characteristics - Colonies on MEA, reaching $2.5 \mathrm{~cm}$ diam. after 2 weeks at 25$30^{\circ} \mathrm{C}$, producing dense mycelium, circular, velvety to woolly, rough margin, white to grey becoming greenish grey when mature, with aerial mycelium. Colonies on PDA reaching $1 \mathrm{~cm}$ diam. after 2 weeks at $25-30^{\circ} \mathrm{C}$, producing dense mycelium, rough margin, white to dark brown to black, with aerial mycelium.

Material examined - RUSSIA, Rostov region, Shakhty City, shrubs near a former coal mine "Proletarian Dictature", on dead twigs of Ulmus pumila (Ulmaceae), 24 March 2016, T.S. Bulgakov, T-1358 (MFLU 16-1652, holotype), ex-type living culture MFLUCC 16-1172 
(asexual morph), ex-type living culture MFLUCC 16-1173 (sexual morph); ibids., T-1295 (MFLU 16-1589), living culture MFLUCC 16-1158; T-1357 (MFLU 16-1651), living culture MFLUCC 16-1171; Rostov region, Shakhty City, Alexandrovsky Park, on dead twigs of Ulmus pumila (Ulmaceae), 14 March 2016, T.S. Bulgakov, T-1326 (MFLU 16-1620), living culture MFLUCC 16-1161; ibids., T-1327 (MFLU 16-1621), living culture MFLUCC 16-1162; T-1328 (MFLU 16-1622), living culture MFLUCC 16-1163; T-1329 (MFLU 16-1623), living culture MFLUCC 16-1165; T-1330 (MFLU 18-0627), living culture MFLUCC 16-1167; T-1331 (MFLU 16-1625), living culture MFLUCC 16-1168, MFLUCC 16-1169; Rostov region, Krasnosulinsky District, Donskoye forestry, artificial forest, on dead twigs of Ulmus pumila (Ulmaceae), 6 April 2016, T.S. Bulgakov, T-1454 (MFLU 16-1748), living culture MFLUCC 16-1179; ibids., T-1455 (MFLU 16-1749), living culture MFLUCC 16-1181; T-1459 (MFLU 16-1749), living culture MFLUCC 16-1182.

Host and distribution - Ulmus pumila (Russia).

Notes - In the NCBI BLASTn search of LSU and ITS sequences, Thyrostroma ulmicola is most similar to T. compactum and T. conicola with $99 \%$ and $98 \%$ similarities, respectively. The asexual morph of T. ulmicola is most similar to T. celtidis, but they are different in the length and width of conidia (T. ulmicola, (30-)35-50(-59) $\times(12-) 15-20(-26) \mu \mathrm{m}$ versus $(24-) 27-39(-42)$ $\times 12-19 \mu \mathrm{m}, T$. celtidis). Phylogenetic analyses of a combined LSU, SSU, ITS and TEF1- $\alpha$ sequence dataset indicated that T. ulmicola forms an independent subclade in Thyrostroma (Figs 2, 3; clade A). In a comparison of ITS and TEF1- $\alpha$ nucleotides, T. ulmicola differs from T. celtidis in 11/480 bp (2.29\%) and 20/822 bp (2.43\%), respectively.

In this study, the sexual morph of Thyrostroma ulmicola is morphologically similar to Dothidotthia species in the shape of asci and ascospores (Ramaley 2005, Phillips et al. 2008, Hyde et al. 2013). However, the peridium structure of T. ulmicola is different from Dothidotthia species. Dothidotthia species have a thin peridium with only 3-6 pigmented cell layers and lack hyaline cell layers, while Thyrostroma has a thick peridium with several hyaline and pigmented cell layers. Furthermore, the ascospores of T. ulmicola are constricted at the septum, whereas ascospores of Dothidotthia species are not constricted or slightly constricted at the septum (Barr 1989).

Thyrostroma ulmigenum Senwanna, Wanas., Bulgakov, Phookamsak \& K.D. Hyde, sp. nov.

Fig. 13

Index Fungorum number: IF556534; Facesoffungi number: FoF06147

Etymology - Named after the host genus Ulmus, from which this species was isolated.

Holotype - MFLU 16-1624

Associated with canker on bark, branches and twigs of Ulmus pumila (Ulmaceae). Sexual morph: Undetermined. Asexual morph: Colonies 100-750 $\mu \mathrm{m}$ diam. sporodochia, stromatic, with pseudoparenchymatous basal stroma, partly immersed to erumpent, rounded to irregularly dehiscent, applanate, black, velvety. Conidiophores (15-)25-67(-77) $\times 3-9 \mu \mathrm{m}) \bar{x}=47 \times 5.7$ $\mu \mathrm{m}, n=70$ ), compactly arranged, macronematous, septate, branched, hyaline to pale brown, smooth. Conidiogenous cells 3-29 $\mu \mathrm{m}$ long, monoblastic, annellidic, with 1-2 annellations, integrated, terminal. Conidia (39-)42-67(-90) $\times(10-) 13-18 \mu \mathrm{m}) \bar{x}=52 \times 14.8 \mu \mathrm{m}, n=80$ ), acrogenous, oblong to subcylindric-clavate, or clavate, phragmosporous to muriform, pale to dark brown, truncate at the base, (3-)4-5(-6) $\mu \mathrm{m}$ diam., rounded at the apex, 3-7 transverse septa, with 0-3 longitudinal septa, constricted at the septa, rough-walled, minutely echinulate.

Culture characteristics - Colonies on MEA, reaching $3 \mathrm{~cm}$ diam. after 2 weeks at $25-30^{\circ} \mathrm{C}$, producing dense mycelium, rough margin, light greenish brown at the centre, dark greenish brown and white at the margin, lacking aerial mycelium. Colonies on PDA, reaching 2 $\mathrm{cm}$ diam. after 3 weeks at $25-30^{\circ} \mathrm{C}$, producing dense mycelium, flat, surface dull and smooth, velvety, rough margin, dark greenish-brown at the centre, white at the margin, lacking aerial mycelium. 
Material examined - RUSSIA, Rostov region, Shakhty Park, Alexandrovsky Park, on fallen and attached twigs of Ulmus pumila (Ulmaceae), 14 March 2016, T.S. Bulgakov, T-1330A (MFLU 16-1624, holotype), ex-type living culture MFLUCC 16-1166; ibid., T-1328B (MFLU 18-0629), living culture, MFLUCC 16-1164.

Host and distribution - Ulmus pumila (Russia).

Notes - In the NCBI BLASTn search of LSU sequences, Thyrostroma ulmigenum is found most similar to T. conicola and T. compactum with 99\% similarity, whereas the closest hits of the ITS sequences were $98 \%$ similar to T. compactum and $97 \%$ similar to T. conicola. In this study, three novel Thyrostroma species, viz. T. tiliae, T. ulmigenum and T. ulmicola were collected from Ulmus pumila. However, these species can be distinguished based on their conidial characters and phylogenetic analyses. Thyrostroma ulmigenum is morphologically similar to T. tiliae, however, these taxa are different in the width of conidia (Table 4). In multigene phylogenetic analyses, T. ulmigenum is sister to T. tiliae (Fig. 3; clade A). In a comparison of ITS and TEF1- $\alpha$ nucleotides, $T$. ulmigenum and T. celtidis are not significantly different from each other (5/479 bp (1.04 \%( of ITS and 10/787 bp (1.27\%) of TEF1- $\alpha$ ). These two species are different in a comparison of RPB2 and TUB2 nucleotides (55/1060 bp (5.19\%) of RPB2 and 15/342 bp (4.39\%( of TUB2). Therefore, we introduce T. ulmigenum as a novel species in Thyrostroma based on morphological characters and the guidelines of Jeewon \& Hyde (2016).

\section{Discussion}

Phylogenetic analyses of the combined LSU, SSU, ITS and TEF1- $\alpha$ gene dataset in the present study (Figs 2, 3) show that Thyrostroma is distinct from Dothidotthia, and concurs with the taxonomic scheme from a previous study (Marin-Felix et al. 2017). Hence, we segregate Thyrostroma from Dothidotthia based upon multi-gene phylogenetic analyses coupled with morphological characteristics. The status of Thyrostroma is resolved and shows that the asexual morph of Thyrostroma is not related to Dothidotthia. Six genera are accepted in Dothidotthiaceae, viz. Dothidotthia, Mycocentrospora, Phaeomycocentrospora, Pleiochaeta, Thyrostroma and Wilsonomyces.

Neodothidotthia was introduced to accommodate $N$. negundinicola and $N$. negundinis by Crous et al. (2019). Crous et al. (2019) analyzed the phylogenetic relationship of Dothidotthia and Neodothidotthia based on LSU sequence dataset. They treated Neodothidotthia as a distinct genus from Dothidotthia and also synonymized Ramaley's collections [described as Thyrostroma negundinis in Ramaley (2005) as well as Dothidotthia aspera in Phillips et al. (2008)] as a synonym of Neodothidotthia negundinis. The combined LSU, SSU, ITS and TEF1- $\alpha$ gene analyses in the present study shows that Neodothidotthia negundinicola and $N$. negundinis nest with $D$. robiniae and $D$. symphoricarpi (Figs 2, 3). In addition, a comparison of the conidial morphology also reveals that these genera are congeneric. Therefore, we synonymize Neodothidotthia under Dothidotthia. In our phylogenetic analyses (analyses 2, Fig. 3), Dothidotthia negundinis strain CPC 12928 forms an unstable lineage, separated from other strains of D. negundinis. This isolate (A.W. Ramaley 0403, BPI 871821) was also collected from a different host. However, there are only ITS, LSU and SSU sequences obtained for this strain which are not appropriate to distinguish species. Therefore, this strain needs to be revisited based on both reliable genes phylogenetic analysis and morphological characteristics.

Dothidotthia and Thyrostroma species have been reported as plant pathogens causing canker, dieback and leaf spot diseases on twig, branch, bark and leaf in both temperate and tropical countries (i.e. Australia, Italy, Korea, Russia, USA) (Yuan \& Old 1990, Kuz'michev et al. 2001, Mel'nik et al. 2007, Phillips et al. 2008, Bulgakov et al. 2014, Marin-Felix et al. 2017). Host-specificity of these taxa have not yet been sorted out and they have been recorded from various plant families, i.e. Asteraceae, Caprifoliaceae, Cannabaceae, Celastraceae, Cornaceae, Compositae, Fabaceae, Hydrangeaceae, Moraceae, Myrtaceae, Oleaceae, Rosaceae, Sapindaceae, Solanaceae and Ulmaceae (Yuan \& Old 1990, Ramaley 2005, Crous et al. 2016, 
Marin-Felix et al. 2017, Farr \& Rossman 2019). More taxon-sampling from other hosts and regions is required for a better understanding of their host-specificity and pathogenicity.

In this study, most species in Dothidotthia and Thyrostroma are quite different from one another based on a comparison of ITS and TEF1- $\alpha$ gene regions. We witnessed that concatenating RPB2 and TUB2 (in primary analyses of our new isolates) provided a good resolution regarding relationships. Therefore, we suggest that RPB2 and TUB2 are reliable genes for distinguishing species within Dothidotthia and Thyrostroma. However, there are mostly ITS, LSU and TEF1- $\alpha$ sequences available in GenBank for Dothidotthia and Thyrostroma from previous studies. Hence, more reliable genes (i.e. RPB2 and TUB2) from further collections, as well as the re-sequencing of the generic types of genera in Dothidotthiaceae are required.

Thyrostroma ulmicola (MFLU 16-1652) forms a holomorph on Ulmus pumila (Fig. 11). Single spore isolation for both isolates (MFLUCC 16-1172 and MFLUCC 16-1173) was obtained from the molecular data. Phylogenetic analyses based on multi-gene sequence data indicated these isolates from sexual and asexual morphs are conspecific and are distinct from Dothidotthia (Figs 2, 3; clade A and D). This is the first record of the sexual morph of Thyrostroma. Thyrostroma ulmicola was collected from symptomatic twigs in European Russia. Recent molecular analyses indicate that eight strains of Thyrostroma ulmicola are separated into two subclades with good support (88\% ML, 88\% MP, 0.99 PP (Fig. 2) and 87\% ML, 90\% MP, 1.00 PP (Fig. 3; clade A), although the conidial characters of these isolates are the same. These isolates have very few morphologically differences and narrow in the host range. Thus, we compared the ITS, RPB2, TEF1- $\alpha$ and TUB2 alignments of these eight strains and found that they are not different. Therefore, we consider these eight strains as the same species based on the morphological similarity of the asexual morph characters. Thyrostroma species are well-known as plant pathogens occurring on twigs and branches (Yuan \& Old 1990, Kuz'michev et al. 2001, Phillips et al. 2008, Crous et al. 2016, Marin-Felix et al. 2017). More collections from different hosts may further clarify their taxonomy and their possibility of occurring as a species complex. The data of Thyrostroma and Dothidotthia provided in this study are a preliminary and more exhaustive sampling of other hosts from other regions will assist in clarifying the taxonomy and the sexual and asexual relationships, as well as, host range and distribution of these taxa.

The genera Mycocentrospora, Pleiochaeta and Wilsonomyces form well-resolved clades in Dothidotthiaceae (Figs 2, 3; clade B, C and D). Mycocentrospora and Pleiochaeta possess unique characteristics derived from Wilsonomyces, due to its appendage at the conidial apex (Pollack \& Ellett 1974, Crous et al. 2013, Marin-Filix et al. 2017). Currently Pleiochaeta comprises six species and only three species are supported by molecular data (Marin-Filix et al. 2017). In our preliminary analyses, Pleiochaeta sp. (B17_3; FJ378717) forms a separate lineage, away from $P$. carotae (type species), however, the strains were obtained from ectomycorrhizal root tips and identified by blast searching of GenBank using only ITS sequence data (Gao \& Yang 2010). We, therefore, did not include this sequence data in our analyses as we cannot verify these data using morphology. Further study of the taxon is needed to resolve its natural placement.

Sutton (1997) treated Wilsonomyces as a synonym of Thyrostroma. However, our phylogenetic analyses showed that $W$. carpophilus (clade B) forms a robust clade distinct from T. compactum (type species of Thyrostroma). Hence, the generic synonymy of Wilsonomyces with Thyrostroma seems unlikely. In addition, Wilsonomyces morphologically resembles Dothidotthia in having similar conidial character, however, its conidia differ in being round to acute at the apex. The genus is phylogenetically distinct from Dothidotthia symphoricarpi, the type species of Dothidotthia. Thus, it is necessary to include more taxa in future studies. 

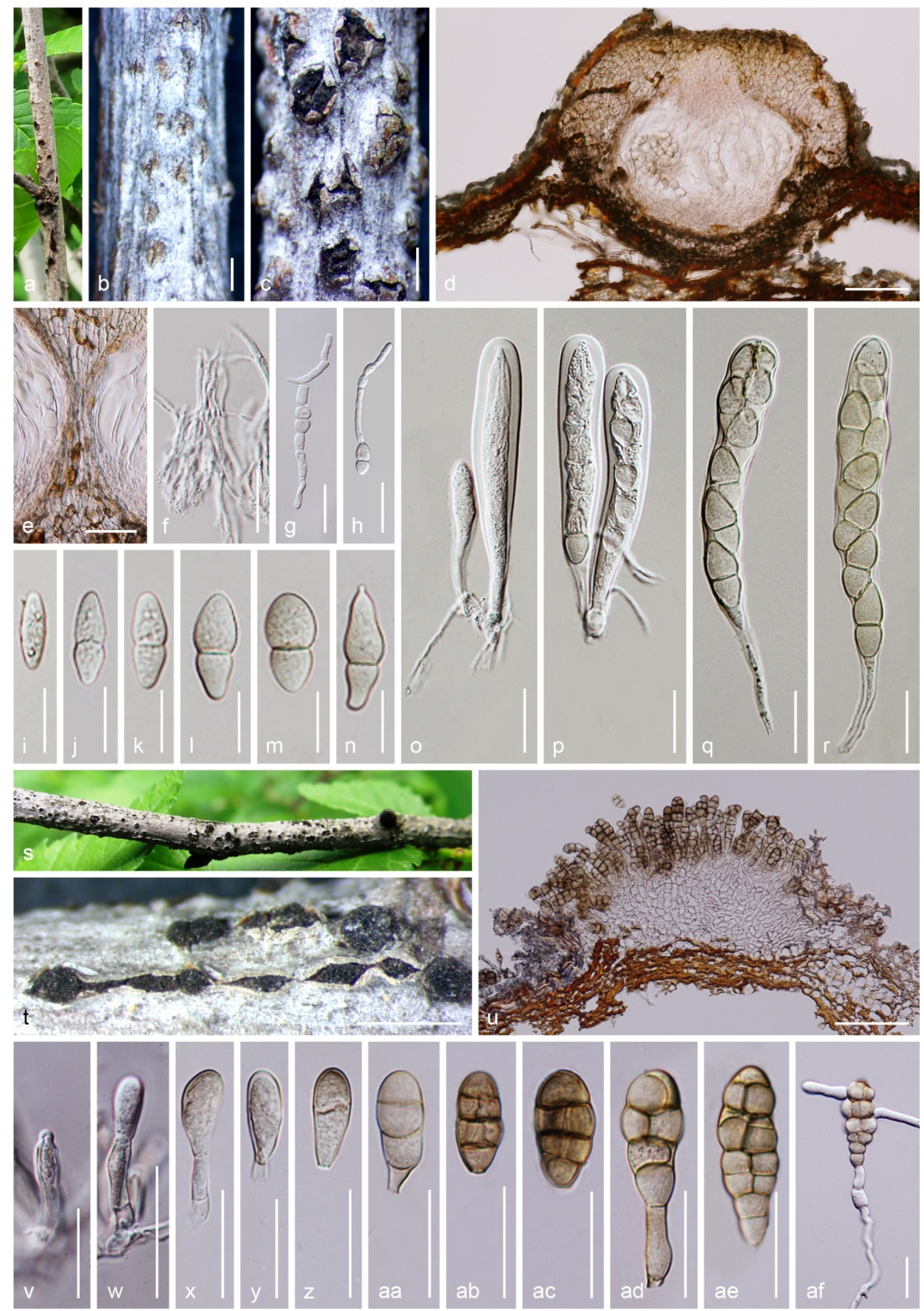

Figure 12 - Thyrostroma ulmicola (MFLU 16-1652, holotype). a-c Ascostromata on bark. d Vertical section of ascoma. e Peridium. f Pseudoparaphyses. g, h Germinated ascospores. i-n Ascospores. o-r Asci. s, t Sporodochia on host surface. u Vertical section of sporodochium. $\mathrm{v}, \mathrm{w}$ Conidiogenesis and conidiogenous cells. $\mathrm{x}$-ae Stages of developing conidia. af Germinated conidium. Scale bars: $\mathrm{b}, \mathrm{c}=500 \mu \mathrm{m}, \mathrm{d}, \mathrm{u}=100 \mu \mathrm{m}, \mathrm{e}=50 \mu \mathrm{m}, \mathrm{f}, \mathrm{i}-\mathrm{r}=30 \mu \mathrm{m}, \mathrm{g}, \mathrm{h}=60 \mu \mathrm{m}$, $\mathrm{t}=1000 \mu \mathrm{m}, \mathrm{v}-\mathrm{af}=20 \mu \mathrm{m}$. 

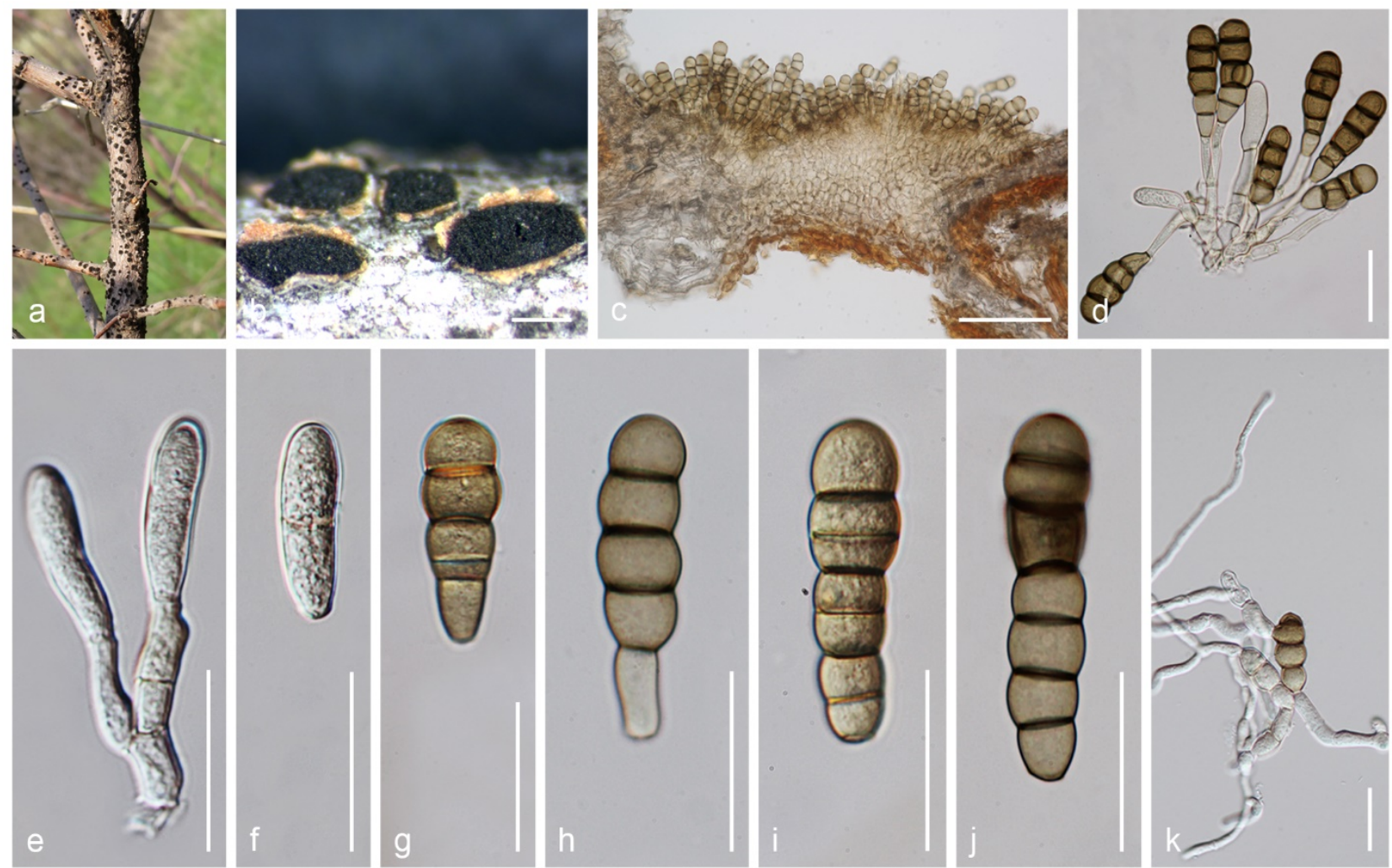

Figure 13 - Thyrostroma ulmigenum (MFLU 18-0629, holotype). a, b Sporodochia on host surface. c Vertical section of sporodochium. d Conidia attached with conidiogenous cells. e Immature conidia attach to conidiogenous cells. $\mathrm{f}-\mathrm{g}$, $\mathrm{i}-\mathrm{j}$ Stages of developing conidia. $\mathrm{h}$ conidia attach with conidiogenous cell. k Germinated conidium. Scale bars: $\mathrm{b}=1000 \mu \mathrm{m}$, $\mathrm{c}=100 \mu \mathrm{m}, \mathrm{d}-\mathrm{k}=30 \mu \mathrm{m}$.

Mycocentrospora was established by Deighton (1972) with $M$. acerina (R. Hartig) Deighton as the type species and classified in Pleosporales genera incertae sedis by Wijayawardene et al. (2018). Phylogenetic analysis based on the LSU sequence data of Dothideomycetes by Crous et al. (2019) shows that M. acerina (MH868490) clustered between Pleiochaeta and Wilsonomyces and similar results are obtained in our study (Figs 1, 2). Mycocentrospora is morphologically similar to Pleiochaeta in having conidia with appendages (Braun 1993, Crous et al. 2013) and is similar to Phaeomycocentrospora in conidial shape (Crous et al. 2013). Species of this genus can be pathogens on living plants and humans and have also been found as saprobes in terrestrial habitats or aquatic hyphomycetes worldwide (Hermansen et al. 1999, Stewart et al. 1999, Wijayawardene et al. 2018). This genus is poorly studied and only sequences of $M$. acerina are available in GenBank. There are 12 epithets of Mycocentrospora listed in Index Fungorum (2019), but four species were accepted by Wijayawardene et al. (2018). More sampling of taxa in this genus is needed for a better understanding.

Previous phylogenetic studies showed that Phaeomycocentrospora belongs in Didymellaceae (Trakunyingcharoen et al. 2014). However, phylogenetic analyses in the present study show that Phaeomycocentrospora (Figs 2, 3; clade F) forms a well-resolved clade in Dothidotthiaceae concurring with Marin-Felix et al. (2017). Even though the morphological characters of Phaeomycocentrospora are different from other genera in Dothidotthiaceae in having mononematous conidiophores, the conidiophores are sometimes reduced to conidiogenous cells, with filiform to cylindrical conidia (Crous et al. 2013). There is a putative species, P. cantuariensis (E.S. Salmon \& Wormald) Crous, H.D. Shin \& U. Braun, available in 
Index Fungorum (2019). Thus, it is necessary to study more taxa coupled with analyses of informative genes for a better understanding of their relationships in the family Dothidotthiaceae.

\section{Acknowledgements}

We would like to thank the Mushroom Research Foundation for partially supporting this work. We acknowledge the Key Research Program of Frontier Sciences of the Chinese Academy of Sciences (grant no. QYZDY-SSW-SMC014) for supporting this research. R. Phookamsak expresses appreciation to the CAS President's International Fellowship Initiative (PIFI) for young staff (grant no. 2019FYC0003), the Yunnan Provincial Department of Human Resources and Social Security (grant no. Y836181261), and National Science Foundation of China (NSFC) project code 31850410489 for financial support. K.D. Hyde would like to thank Thailand Research Fund )TRF( grant no DGB6080013 entitled "The future of specialist fungi in a changing climate: baseline data for generalist and specialist fungi associated with ants, Rhododendron species and Dracaena species”. D.N. Wanasinghe would like to thank CAS President's International Fellowship Initiative (PIFI number 2019PC0008) and the 64th batch of China Postdoctoral Science Foundation (grant no.: Y913083271) for funding his postdoctoral research. T.S. Bulgakov would like to thank K.D. Hyde for the support of mycological researches in Russia. Peter E. Mortimer and D.N. Wanasinghe thank the National Science Foundation of China and the Chinese Academy of Sciences for financial support under the following grants: 41761144055, 41771063 and Y4ZK111B01. Y. Wang would like to thank the project of National Natural Science Foundation of China (No. 31560489), Talent project of Guizhou science and technology cooperation platform ([2017]5788-5) and Guizhou science, technology department international cooperation base project ([2018]5806) for carrying out molecular work. Dr. Shaun Pennycook is thanked for his essential nomenclatural review. We thank Prof. Dr. Eric H.C. McKenzie, Asst. Prof. Dr. Ratchadawan Cheewangkoon, Dr. Saowaluck Tibpromma, Sirinapa Konta, Qiu-Ju Shang and Milan C. Samarakoon for their valuable suggestions and help in phylogenetic analyses.

\section{References}

Ariyawansa HA, Phillips AJL, Chuang W-Y, Tsai I. 2018 - Tzeananiaceae, a new pleosporalean family associated with Ophiocordyceps macroacicularis fruiting bodies in Taiwan. MycoKeys 37: 1-17.

Ariyawansa HA, Phukhamsakda C, Thambugala KM, Bulgakov TS et al. 2015a - Revision and phylogeny of Leptosphaeriaceae. Fungal Diversity 74, 19-51.

Ariyawansa HA, Thambugala KM, Manamgoda DS, Jayawardena R et al. 2015b - Towards a natural classification and backbone tree for Pleosporaceae. Fungal Diversity 71, 85-139.

Barr ME. 1989 - The Genus Dothidotthia (Botryosphaeriaceae) in North America. Mycotaxon 2, 517-526.

Bulgakov TS, Vasilyev NP, Zmitrovich IV. 2014 - Summarizing of 10-years investigation on mycobiota of alien trees and shrubs in arboretum of the Otradnoye Research Station of the Komarov Botanical Institute. Botany: history, theory, practice (on the occasion of the 300th anniversary of the founding of the V.L. Komarov Botanical Institute of the Russian Academy of Sciences). V.L. Komarov Botanical Institute, St. Petersburg, Russia: 31-39 (in Russian).

Braun U. 1993 - Taxonomic notes on some species of the Cercospora complex (III). Mycotaxon 48, 275-298.

Chen Q, Hou LW, Duan WJ, Crous PW, Cai L. 2017 - Didymellaceae revisited. Studies in Mycology 87, 105-59.

Crous PW, Braun U, Hunter GC, Wingfeld MJ et al. 2013 - Phylogenetic lineages in Pseudocercospora. Studies in Mycology 75, 37-114. 
Crous PW, Wingfield MJ, Richardson DM, Le Roux JJ et al. 2016 - Fungal Planet description sheets: 400-468. Persoonia 36, 316-458.

Crous PW, Schumache RK, Akulov A, Thangavel R et al. 2019 - New and Interesting Fungi. 2. Fungal Systematcs and Evolution 3, 57-134.

Crous PW, Slippers B, Wingfield MJ, Rheeder J et al. 2006 - Phylogenetic lineages in Botryosphaeriaceae. Studies in Mycology 55, 235-253.

Deighton FC. 1972 - Mycocentrospora, a new name for Centrospora Neerg. Taxon 21, 716-716.

Farr DF, Rossman AY. 2019 - Fungal Databases, U.S. National Fungus Collections, ARS, USDA. https://nt.ars-grin.gov/fungaldatabases/ (Accessed January to March 2019).

Felsenstein J. 1985 - Confidence limits on phylogenies: An approach using the bootstrap. Evolution 39, 783-791.

Gao Q, Yang ZL. 2010 - Ectomycorrhizal fungi associated with two species of Kobresia in an alpine meadow in the eastern Himalaya. Mycorrhiza 20, 281-287.

Glass NL, Donaldson GC. 1995 - Development of primer sets designed for use with the PCR to amplify conserved genes from filamentous ascomycetes. Applied and Environmental Microbiology 61, 1323-1330.

Hall TA. 1999 - BioEdit: a user-friendly biological sequence alignment editor and analysis program for Windows 95/98/NT. Nucleic Acids Symposium 41, 95-98.

Hermansen A, Amundsen T, Taksdal G, Dragland S et al. 1999 - Variations in infection by Mycocentrospora acerina in carrot monoculture plots at four sites during 1985-1995. Acta Agriculturae Scandinavica, Section B - Soil \& Plant Science 49, 248-257.

Höhnel FXR von. 1911 - Fragmente zur Mykologie. XIII Mitteilung (Nr. 642 bis 718). Sitzungsberichte der Kaiserlichen Akademie der Wissenschaften in Wien MathematischNaturwissenschaftliche Classe, Abt. 1 120, 379-484.

Huelsenbeck JP, Ronquist F. 2001 - MRBAYES: Bayesian inference of phylogenetic trees. Bioinformatics 17, 754-755.

Hyde KD, Jones EBG, Liu JK, Ariyawansa HA et al. 2013 - Families of Dothideomycetes. Fungal Diversity 63, 1-313.

Index Fungorum. 2019 - Index Fungorum. Available from: http://www.indexfungorum.org/ Names/Names.asp Accessed May 2019.

Jaklitsch WM, Checa J, Blanco MN, Olariaga I et al. 2018 - A preliminary account of the Cucurbitariaceae. Studies in Mycology 90, 71-118.

Jayasiri SC, Hyde KD, Ariyawansa HA, Bhat J et al. 2015 - The Faces of Fungi database: fungal names linked with morphology, phylogeny and human impacts. Fungal Diversity 74, 3-18.

Jeewon R, Hyde KD. 2016 - Establishing species boundaries and new taxa among fungi: recommendations to resolve taxonomic ambiguities. Mycosphere 7, 1669-1677.

Katoh K, Rozewicki J, Yamada KD. 2017 - MAFFT online service: multiple sequence alignment, interactive sequence choice and visualization. Briefings in Bioinformatics.

Kishino H, Hasegawa M. 1989 - Evaluation of the maximum likelihood estimate of the evolutionary tree topologies from DNA sequence data, and the branching order in Hominoidea. Journal of Molecular Evolution 29, 170-179.

Kolemasova NN. 1999 - Dendrotrophic mycobiota and phytopathological state of parks of Pushkin and Pavlovsk towns. Forestry Bulletin 2, 68-69 (in Russian).

Kolganikhina GB, Sokolova ES. 2012 - The most important fungal diseases of trees and shrubs in green plantings of Moscow and Moscow suburbs. Forestry Ideas 18(1), 97-103 (in Russian).

Kumar S, Stecher G, Tamura K. 2015 - MEGA7: Molecular Evolutionary Genetics Analysis version 7.0 for bigger datasets. Molecular Biology and Evolution 33, 1870-1874.

Kuz'michev EP, Sokolova ES, Kulikova EG. 2001 - Common Fungal Diseases of Russian Forests. Gen. Tech. Rep. NE-279. Newtown Square, PA; U.S Department of Agriculture, Forest Service, Northeastern Research Station. 137p. 
Liu JK, Hyde KD, Jeewon R, Phillips AJL et al. 2017 - Ranking higher taxa using divergence times: a case study in Dothideomycetes. Fungal Diversity 84, 75-99.

Liu NG, Ariyawansa HA, Hyde KD, Maharachchikumbura SSN et al. 2016 - Perspectives into the value of genera, families and orders in classification. Mycosphere 7, 1649-1668.

Liu YJ, Whelen S, Hall BD. 1999 - Phylogenetic relationships among ascomycetes: evidence from an RNA polymerse II subunit. Molecular Biology and Evolution 16, 1799-1808.

Marin-Felix Y, Groenewald JZ, Cai L, Chen Q et al. 2017 - Genera of phytopathogenic fungi: GOPHY 1. Studies in Mycology 86, 99-216.

Mel'nik VA, Popov ES, Shabunin DA. 2007 - Contributions to the studies of mycobiota in Novgorod and Pskov regions. I. Hyphomycetes. Mikologia i Fitopatologia 41, 515-525. (in Russian)

Miller MA, Pfeiffer W, Schwartz T. 2010 - Creating the CIPRES science gateway for inference of large phylogenetic trees. Proceedings of the Gateway Computing Environments Workshop (GCE) 1, 1-8.

Morgan-Jones G. 1971 - Sciniatosporium Kalchbr., and its synonyms Mavcosia Syd., Stigmina Sacc., Thyrostroma Hohnel, and Thyrostromella Syd., non Hijhnel. Canadian Jpurnal of Botany 49, 993-1009.

Pem D, Jeewon R, Bulgakov T, Gafforov Y et al. 2019 - Taxonomy and molecular phylogeny of Thyrostroma ephedricola sp. nov. and proposal for Thyrostroma jaczewskii comb. nov. Phytotaxa 416, 243-256.

Phillips AJL, Alves A, Pennycook SR, Johnston PR et al. 2008 - Resolving the phylogenetic and taxonomic status of dark-spored teleomorph genera in the Botryosphaeriaceae. Persoonia 21, 29-55.

Phookamsak R, Liu JK, McKenzie EHC, Manamgoda DS et al. 2014 - Revision of Phaeosphaeriaceae. Fungal Diversity 68, 159-238.

Phookamsak R, Wanasinghe DN, Hongsanan S, Phukhamsakda C et al. 2017 - Towards a natural classification of Ophiobolus and ophiobolus-like taxa; introducing three novel genera Ophiobolopsis, Paraophiobolus and Pseudoophiobolus in Phaeoshaeriaceae (Pleosporales). Fungal Diversity 87, 299-339.

Pollack FG, Ellett CW. 1974 - Mycocentrospora verrucosa, the Cause of Foliar Shot-Hole of Euonymus. Mycologia 66, 170-173.

Potebnia A. 1907 - Mycologische Studien. Annales Mycologici 5, 1-28.

Ramaley AW. 2005 - The connection of Dothidotthia aspera (Botryosphaeriaceae) to a hyphomycetous anamorphic fungus, Thyrostroma negundinis. Mycotaxon 94, 127-132.

Rambaut A. 2016 - FigTree, version 1.4.3. University of Edinburgh, Edinburgh.

Rambaut A, Suchard MA, Xie D, Drummond AJ. 2013 - MCMC Trace Analysis Tool. Version v1.6.0. Available from: http://beast.bio.ed.ac.uk/Tracer. Accessed 03 March 2019.

Rannala B, Yang Z. 1996 - Probability distribution of molecular evolutionary trees: a new method of phylogenetic inference. Journal of Molecular Evolution 43, 304-311.

Rehner SA. 2001 - Primers for elongation factor 1-alpha (EF1-alpha). Available from: http://ocid.nacse.org/research/deephyphae/EF1primer.pdf. Accessed December 2018.

Slippers B, Boissin E, Phillips AJL, Groenewald JZ et al. 2013 - Phylogenetic lineages in the Botryosphaeriales: a systematic and evolutionary framework. Studies in Mycology 76, 3149.

Sokolova ES. 2003 - Stigmina cancer disease of elms in urban plantings. Forestry bulletin 27(2), 74-77 (in Russian).

Sokolova ES, Kolganikhina GB, Galasyeva TV, Strepanyuk LP et al. 2006 - Species composition and distribution of xylophagous fungi in different green stands of Moscow. Forestry Bulletin 44 (2), 98-116 (in Russian).

Stamatakis A. 2014 - RAxML version 8: a tool for phylogenetic analysis and post-analysis of large phylogenies. Bioinformatics 30, 1312-1313. 
Stamatakis A, Hoover P, Rougemont J. 2008 - A rapid bootstrap algorithm for the RAxML web servers. Systematic Biology 57, 758-771.

Stewart EL, Liu Z, Crous PW, Szabo LJ. 1999 - Phylogenetic relationships among some cercosporoid anamorphs of Mycosphaerella based on rDNA sequence analysis. Mycological Research 103, 1491-1499.

Stravinskienė V, Snieškienė V, Stankevičienè A. 2015 - Health condition of Tilia cordata Mill. trees growing in the urban environment. Urban Forestry \& Urban Greening 14(1), 115122.

Sutton BC. 1997 - On Stigmina, Wilsonomyces and Thyrostroma (Hyphomycetes). In: Arnoldia 14, 33-35.

Sutton BC, Pascoe IG. 1989 - Reassessment of Peltosoma, Stigmina and Batcheloromyces and description of Hyphothyrium gen. nov. Mycological Research 92, 210-222.

Swofford DL. 2002 - PAUP*: phylogenetic analysis using parsimony (*and other methods). Version 4. Sinauer Associates, Sunderland.

Trakunyingcharoen T, Lombard L, Groenewald JZ, Cheewangkoon R et al. 2014 Mycoparasitic species of Sphaerellopsis, and allied lichenicolous and other genera. IMA Fungus 5, 391-414.

Valenzuela-Lopez N, Cano-Lira JF, Guarro J, Sutton DA et al. 2018 - Coelomycetous Dothideomycetes with emphasis on the families Cucurbitariaceae and Didymellaceae. Studies in Mycology 90, 1-69.

Vilgalys R, Hester M. 1990 - Rapid genetic identification and mapping of enzymatically amplified ribosomal DNA from several Cryptococcus species. Journal of Bacteriology 172, 4238-4246.

Wanasinghe DN, Hyde KD, Jeewon R, Crous PW et al. 2017a - Phylogenetic revision of Camarosporium (Pleosporineae, Dothideomycetes) and allied genera. Studies in Mycology 87, 207-256.

Wanasinghe DN, Jeewon R, Jones EBG, Boonmee S et al. 2018 - Novel palmicolous taxa within Pleosporales: Multigene phylogeny and taxonomic circumscription. Mycological Progress 17, 571-590.

Wanasinghe DN, Phookamsak R, Jeewon R, Li WJ, et al. 2017b - Fenestellaceae with descriptions of new Fenestella species and Neocucurbitaria gen. nov. Mycosphere 8, 397414.

White T, Bruns T, Lee S, Taylor J. 1990 - Amplification and direct sequencing of fungal ribosomal RNA genes for phylogenetics. In: Innis, M., Gelfand, D., Shinsky, J. \& White, T. (Eds.) PCR protocols: a guide to methods and applications. Academic Press, New York, 315-322 pp.

Wijayawardene NN, Crous PW, Kirk PM, Hawksworth DL et al. 2014 - Naming and outline of Dothideomycetes-2014 including proposals for the protection or suppression of generic names. Fungal Diversity 69, 1-55.

Wijayawardene NN, Hyde KD, Lumbsch HT, Liu JK et al. 2018 - Outline of Ascomycota: 2017. Fungal Diversity 88, 167-263.

Yuan ZQ, Old KM. 1990 - A new species of Thyrostroma from Australia. Mycological Research 94, 573-576.

Zhang H, Hyde KD, Mckenzie EHC, Bahkali AH, Zhou DQ. 2012 - Sequence data reveals phylogenetic affinities of Acrocalymma aquatica sp. nov., Aquasubmersa mircensis gen. et sp. nov. and Clohesyomyces aquaticus (freshwater coelomycetes). Cryptogamie Mycologie 33, 333-346.

Zhaxybayeva O, Gogarten JP. 2002 - Bootstrap, Bayesian probability and maximum likelihood mapping: exploring new tools for comparative genome analyses. BMC Genomics 3, 1-15. 